Running head: Linguistic distance and psychotherapy

\title{
Linguistic measures of psychological distance track symptom levels and treatment outcomes in a large set of psychotherapy transcripts
}

\author{
Erik C. Nook ${ }^{1}$, Thomas D. Hull ${ }^{2,3}$, Matthew K. Nock ${ }^{1}, \&$ Leah H. Somerville ${ }^{1}$ \\ ${ }^{1}$ Department of Psychology, Harvard University \\ Cambridge, MA 02138 \\ ${ }^{2}$ Talkspace \\ New York, NY 10025 \\ ${ }^{3}$ Department of Counseling and Clinical Psychology, Columbia University \\ New York, NY 10027

\section{THIS IS A PREPRINT ONLY, PLEASE SEE PUBLISHED FINAL VERSION OF RECORD AT https://doi.org/10.1073/pnas.2114737119}

\author{
Address correspondence to: \\ Erik C. Nook \\ Department of Psychology \\ Princeton University \\ Peretsman Scully Hall \\ Princeton, NJ 08540 \\ enook@princeton.edu
}

Author Contributions: ECN and LS designed research questions. TDH provided Talkspace data and insight into therapy platform. ECN, LS, and MN designed analytic approach. ECN processed data and conducted analyses. ECN and LS drafted manuscript. All authors provided revisions and approved of the final submission.

Competing Interest Statement: TDH is an employee of Talkspace. To eliminate impact of this conflict of interest on the study, TDH did not contribute to decisions concerning data analysis or reporting of results. MKN receives publication royalties from Macmillan, Pearson, and UpToDate. MKN has been a paid consultant in the past year for Microsoft Corporation, the Veterans Health Administration, Cerebral, and for a legal case regarding a death by suicide. MKN is an unpaid scientific advisor for Empatica, Koko, and TalkLife. These roles are not perceived as creating conflicts of interests but are reported for transparency. ECN and LHS declare no conflicts of interest.

Classification: Social Sciences / Psychological and Cognitive Sciences.

Keywords: Language, linguistic distance, treatment outcomes, internalizing symptoms, psychotherapy

$\begin{array}{ll}\text { Abstract word count: } & 223 \\ \text { Significance statement word count: } & 100 \\ \text { Main text word count: } & 7,077 \\ \text { Number of tables: } & 1 \\ \text { Number of figures: } & 3 \\ \text { Number of references: } & 86\end{array}$




\begin{abstract}
Using language to distance oneself from negative stimuli (e.g., by reducing use of the word "I" and present-tense verbs) is associated with effective emotion regulation. Given that internalizing disorders like anxiety and depression are characterized by maladaptive emotion regulation, stronger linguistic distance may be both a diagnostic marker of lower internalizing symptoms and a prognostic indicator of treatment progress. Here, we tested these hypotheses in a large corpus of naturalistic psychotherapeutic exchanges between clients and their therapists (> 1.2 million messages from 6,229 clients). In both exploratory $(N=3,729)$ and validation $(N=2,500)$ datasets, we found that clients' internalizing symptoms decreased over therapy, that client linguistic distance increased over therapy, and that internalizing symptoms tracked fluctuations in linguistic distance both within- and between-individuals. In other words, clients shifted from discussing themselves and the present moment to discussing other people and timepoints over treatment, and this psycholinguistic shift was related to symptom reductions. However, effect sizes for linguistic results were small, and we failed to find consistent evidence that linguistic distance statistically mediated changes in symptoms over time. Finally, clustering analyses revealed that data-driven groups of clients defined based solely on their linguistic distance differed in both their symptom severity and treatment outcomes. Together, these findings provide replicable evidence that linguistic distance is a marker of internalizing symptom severity and treatment progress in real-world therapeutic interactions.
\end{abstract}




\section{SIGNIFICANCE STATEMENT}

Using language to "distance" ourselves from distressing situations (i.e., by talking less about ourselves and the present moment) can help us manage emotions. Here we translate this basic research to discover that such "linguistic distancing" is a replicable measure of mental health in a large set of therapy transcripts $(\mathrm{N}=6,229)$. Additionally, clustering techniques showed that language alone could identify participants who differed on both symptom severity and treatment outcomes. These findings lay the foundation for (i) tools that can rapidly identify people in need of psychological services based on language alone and (ii) linguistic interventions that can improve mental health. 


\section{Introduction}

Psychopathology is both common and costly: Approximately 50\% of Americans will experience a psychological disorder and psychopathology accounts for $7 \%$ of the global burden of disease $(1,2)$. Scholars agree that the current mental healthcare system is insufficient for meeting this demand due to a number of factors including there being too few therapists, therapists being hard to reach, and most therapies having only moderate efficacy (3-5). As such, there is dire need for tools that increase treatment accessibility and efficacy. Clinical scientists have called for technological innovations that could achieve these goals (6-9), leading to a wave of technology-assisted psychotherapies in which therapists treat clients via text messaging (1012). These platforms increase the reach of any given therapist and can effectively treat internalizing disorders like anxiety and depression at a fraction of the cost of in-person treatment (11-14). Although these technological innovations provide a vital step towards addressing the burden of global mental health, there remains a need for tools that can detect individuals who may need them, as well as techniques that improve the efficacy of existing treatments. Language represents a prime entry point for developing these detection and intervention tools at a large scale, given the facts that (i) verbal and digital conversation is ubiquitous in human society and (ii) psychotherapeutic interventions are essentially a set of conversations. In this study, we leverage basic understanding of the relationships between language and emotion to demonstrate that a linguistic measure of healthy emotion regulation tracks psychological symptoms in a large set of psychotherapy transcripts.

Every day, people use words to identify and communicate about their emotional experiences (15-17). Difficulties with emotion regulation (i.e., the ways in which people modify or manage their emotional experiences $[18,19])$ are robustly connected to psychopathology (20- 
22), and successful therapy operates through changing patients' emotion regulation habits (2326). Consequently, finding a linguistic signature of poor emotion regulation could measure levels of psychopathology and their remission over treatment at large scale. Interestingly, a growing number of studies show that using language to increase psychological distance (i.e., "taking a step back" and seeing challenging situations as separated from oneself [27, 28]) can facilitate effective emotion regulation (29-33). Increasing distance along "social" and "temporal" dimensions by reducing use of first-person singular pronouns (e.g., "I") and present-tense verbs (e.g., "feel") both decreases the intensity of negative affect and predicts the success of emotion regulation $(29,30)$.

These studies provide compelling evidence that linguistic distance predicts emotion regulation success in the lab, and here we ask about its clinical relevance in real-world therapeutic conversations. Individuals who fail to gain distance from their negative emotions may struggle to effectively regulate these emotions, leading to symptoms of internalizing disorders like anxiety and depression. If so, linguistic markers of low psychological distance should track symptoms of psychopathology. Preliminary results align with this notion, as greater use of first-person singular pronouns (indicating less psychological distance along the social dimension) is associated with clinical problems like depression, anxiety, PTSD, and suicide (3440). However, only a handful of studies have investigated this relationship within the context of treatment itself, and they have returned mixed results (41-44). Consequently, there is a need for a large, systematic test of whether linguistic distance is longitudinally associated with psychological symptoms in naturalistic exchanges during psychotherapy.

Here, we investigate relations between linguistic distance and psychotherapy outcomes in a large dataset ( $N=6,229$ participants) from a message-based psychotherapy service (Talkspace). 
Talkspace clients and their licensed therapists communicate primarily via text messaging, and clients complete self-report inventories of anxiety and depression symptoms every three weeks. In this study, we examined a programmatic set of research questions that together test whether a client's linguistic distance tracks levels of internalizing symptoms both between- and withinindividuals and whether it might serve as a mediating mechanism of treatment outcomes. We conducted analyses initially in an exploratory subset of the data $(N=3,727)$. We then preregistered our hypothesized model and analytic plan (https://osf.io/r5gn2) and replicated all analyses in a holdout validation dataset $(N=2,500)$. Our analyses investigated (i) simple linear relations between time in treatment, symptoms, and linguistic distance; (ii) whether increasing linguistic distance mediated reduced symptoms over time in treatment, and (iii) whether clusters of clients defined purely on the trajectory of their linguistic distance over treatment differed in their symptom severity or treatment outcomes. Establishing replicable relations between linguistic distance and symptoms in such large-scale real-world data lays a foundation for research that can use language to both detect people at risk for psychopathology and enhance the efficacy of psychotherapy, ultimately reducing the global burden of psychopathology.

\section{Results}

\section{Are Internalizing Symptoms, Linguistic Distance, and Time in Treatment Related?}

Internalizing symptoms over time in treatment. Mixed-effects regressions showed that internalizing symptoms fell over the course of treatment with a medium effect size in both the exploratory and validation datasets, $\beta_{\mathrm{e}}=-.42, p_{\mathrm{e}}<.001, R_{\beta \mathrm{e}}^{2}=.37, \beta_{\mathrm{v}}=-.43, p_{\mathrm{v}}<.001, R_{\beta \mathrm{v}}^{2}=.40($ Figure 1). The subscripts "e" and " $v$ " are used to indicate that statistics are from the exploratory and validation datasets, respectively. 
Linguistic distance over time. Linguistic distance (i.e., client's use of verbs and pronouns that were distanced from themselves and the present moment) increased over time in therapy in both the exploratory and validation datasets, $\beta_{\mathrm{e}}=.07, p_{\mathrm{e}}<.001, R_{\beta \mathrm{e}}^{2}=.02, \beta_{\mathrm{v}}=.08$, $p_{\mathrm{v}}<.001, R_{\beta \mathrm{v}}^{2}=.02$ (Figure 1). A small effect size indicated that this was a subtle linguistic shift over the course of therapy.

Linguistic distance and internalizing symptoms. We observed small but significant relationships between internalizing symptoms and linguistic distance in the exploratory and validation datasets, $\beta_{\mathrm{e}}=-.12, p_{\mathrm{e}}<.001, R_{\beta \mathrm{e}}^{2}=.05, \beta_{\mathrm{v}}=-.14, p_{\mathrm{v}}<.001, R_{\beta \mathrm{v}}^{2}=.06$. As hypothesized, worse internalizing symptoms were associated with less distanced language. We next decomposed variance in linguistic distance into within-person and between-person components (Figure 1). Mixed-effects models revealed that internalizing symptoms were significantly associated with both between-person, $\beta_{\mathrm{e}}=-.20, p_{\mathrm{e}}<.001, R_{\beta \mathrm{e}}^{2}=.03, \beta_{\mathrm{v}}=-.21, p_{\mathrm{v}}<.001, R_{\beta \mathrm{v}}^{2}=.04$, and within-person, $\beta_{\mathrm{e}}=-.04, p_{\mathrm{e}}<.001, R_{\beta \mathrm{e}}^{2}=.02, \beta_{\mathrm{v}}=-.06, p_{\mathrm{v}}<.001, R_{\beta \mathrm{v}}^{2}=.005$, variance in linguistic distance. Effects ranged from very small to small, and effects were larger for between-person than within-person relationships.

\section{Does Linguistic Distance Mediate Symptom Reduction?}

Mediation analyses implemented in a Bayesian framework produced mixed support for the notion that within-person increases in linguistic distance mediate decreased symptoms across time. The mediation model was not significant in the exploratory dataset (Figure 2a), but it was significant in the validation dataset (Figure 2b). The very small proportion mediated in the validation dataset $(0.3 \%)$ indicates that the potential mediating role of within-person fluctuations in internalizing symptoms is extremely small. 


\section{Can Symptoms be Inferred from Linguistic Patterns Alone?}

We used finite mixture regression analyses to cluster clients into groups that shared similar trajectories of linguistic distance over treatment. We then found that these groupsdefined based on language alone - significantly differed in both treatment outcomes and symptom severity. We first established that a four-cluster mixture regression solution provided the best fit to the data for both the exploratory dataset $\left(\mathrm{AIC}_{\mathrm{e}-4 \text {-cluster }}=3,562,482 ; \mathrm{AIC}_{\mathrm{e}-3 \text { - }}\right.$ cluster $\left.=3,567,742 ; \mathrm{AIC}_{\mathrm{e}-2 \text {-cluster }}=3,574,110 ; \mathrm{AIC}_{\mathrm{e}-1 \text {-cluster }}=3,593,658\right)$ and the validation dataset $\left(\mathrm{AIC}_{\mathrm{v}-4-\text { cluster }}=2,388,523 ; \mathrm{AIC}_{\mathrm{v}-3 \text {-cluster }}=2,392,075 ; \mathrm{AIC}_{\mathrm{v}-2 \text {-cluster }}=2,395,775 ; \mathrm{AIC}_{\mathrm{v}-1 \text { - }}\right.$ cluster $=2,411,255)$. Clusters were remarkably similar across the exploratory and validation dataset, even though they were defined completely independently (Figures 3a and 3e). Linguistic distance for clusters $1_{\mathrm{e}}$ and $1_{\mathrm{v}}$ started high and rose over therapy (though not significantly for the validation dataset), $\beta_{\mathrm{e}}=.08, p_{\mathrm{e}}=.002, R_{\beta \mathrm{e}}^{2}=.02, N_{\mathrm{e}}=569, \beta_{\mathrm{v}}=.06, p_{\mathrm{v}}=.139, R_{\beta \mathrm{v}}^{2}=.01, N_{\mathrm{v}}=270$. Clusters $2_{\mathrm{e}}$ and $2_{\mathrm{v}}$ started slightly less high but rose strongly over therapy, $\beta_{\mathrm{e}}=.13, p_{\mathrm{e}}<.001, R_{\beta \mathrm{e}}^{2}=.05$, $N_{\mathrm{e}}=1,277, \beta_{\mathrm{v}}=.14, p_{\mathrm{v}}<.001, R_{\beta \mathrm{v}}^{2}=.06, N_{\mathrm{v}}=722$. Clusters $3_{\mathrm{e}}$ and $3_{\mathrm{v}}$ started low and remained low over therapy, $\beta_{\mathrm{e}}=.01, p_{\mathrm{e}}=.610, R_{\beta \mathrm{e}}^{2}=.0004, N_{\mathrm{e}}=735, \beta_{\mathrm{v}}=.02, p_{\mathrm{v}}=.501, R_{\beta \mathrm{v}}^{2}=.001, N_{\mathrm{v}}=654$. Finally, clusters $4_{\mathrm{e}}$ and $4_{\mathrm{v}}$ started low and rose over therapy, $\beta_{\mathrm{e}}=.06, p_{\mathrm{e}}=.001, R_{\beta \mathrm{e}}^{2}=.01, N_{\mathrm{e}}=1,146,{ }^{1} \beta_{\mathrm{v}}=.09$, $p_{\mathrm{v}}<.001, R_{\beta \mathrm{v}}^{2}=.02, N_{\mathrm{v}}=854$.

A one-way ANOVA revealed that these clusters - defined solely based on trajectories of linguistic distance over time-differed significantly in baseline internalizing symptom scores in both the exploratory and validation dataset, $F_{\mathrm{e}}(3,3723)=14.31, p_{\mathrm{e}}<.001, \eta^{2}{ }_{\mathrm{e}}=.01, F_{\mathrm{v}}(3$, 2496) $=9.18, p_{\mathrm{v}}<.001, \eta^{2}=.01$ (Figures 3b and 3f). Pairwise comparisons of conditions revealed

\footnotetext{
${ }^{1}$ Two participants from the exploratory dataset were not included in temporal distance or social distance clusters because they had no text messages that included verbs or pronouns. These participants sent very few text messages (i.e., 13 or fewer).
} 
remarkably consistent results in both the validation and exploratory datasets. Post-hoc Tukey comparisons revealed that baseline internalizing symptom levels were significantly lower for clusters 1 and 2 than clusters 3 and $4, p_{\mathrm{e}} \mathrm{s}<.001, p_{\mathrm{v}} \mathrm{s}<.05$. Clusters 1 and 2 did not differ in their baseline symptom levels, and neither did clusters 3 and $4, p_{\mathrm{e}} \mathrm{s}>.175, p_{\mathrm{v}} \mathrm{s}<.641$. As such, clusters of clients who started treatment with higher linguistic distance had lower internalizing symptoms at baseline.

Analyses of final internalizing symptom scores showed similar patterns. Clusters differed significantly in their final symptom levels in both the exploratory and validation datasets, $F_{\mathrm{e}}(3$, $3723)=23.18, p_{\mathrm{e}}<.001, \eta^{2} \mathrm{e}=.02, F_{\mathrm{v}}(3,2496)=13.94, p_{\mathrm{v}}<.001, \eta^{2}{ }_{\mathrm{v}}=.02$, (Figures 3c and 3g). Tukey post-hoc comparisons indicated that final internalizing symptom scores were significantly lower for clusters 1 and 2 compared to clusters 3 and $4, p_{\mathrm{e}} \mathrm{s}<.001, p_{\mathrm{v}}<<.006$. Again, clusters 1 and $2 \mathrm{did}$ not differ from each other, and neither did clusters 3 and $4, p_{\mathrm{e}} \mathrm{s}>.848, p_{\mathrm{v}} \mathrm{s}<.848$. Again, clusters of clients with higher linguistic distance had less severe internalizing symptoms.

Finally, one-way ANCOVAs showed that these clusters also differed in how strongly their internalizing symptoms changed across therapy (i.e., final - baseline internalizing symptoms scores, controlling for baseline scores $), F_{\mathrm{e}}(3,3722)=5.49, p_{\mathrm{e}}<.001, \eta_{\mathrm{p}}{ }^{2}=.004, F_{\mathrm{v}}(3$, $2495)=2.85, p_{\mathrm{v}}=.036, \eta_{\mathrm{p}}{ }^{2}=.003$ (Figures 3d and 3h). Post-hoc Tukey tests revealed that symptoms fell more strongly across treatment for cluster 2 than for clusters 3 and $4, p_{\mathrm{e}} \mathrm{s}<.001$, $p_{\mathrm{v}}<<.02$. As such, the cluster with the strongest increase in linguistic distance across treatment also had the greatest treatment response. In the validation dataset only, cluster $3_{v}$ also showed significantly less treatment gain than cluster $1_{\mathrm{v}}, p_{\mathrm{v}}=.029$, meaning that the cluster that did not increase in linguistic distance over treatment faired poorest. No other pairwise comparisons for change in internalizing symptoms reached significance, $p_{\mathrm{e}} \mathrm{s}>.07, p_{\mathrm{v}} \mathrm{S}>.31$. As such, clustering 
participants based on trajectories of linguistic distance revealed replicable signatures of participants who differed both in their overall symptom severity and their treatment response. Analyses of temporal and social components of the linguistic distancing measure revealed that temporal distance clusters differed in treatment outcomes but not baseline symptom levels, whereas social distance clusters differed in chronic symptom levels but not treatment outcomes (see Supplementary Information).

\section{Discussion}

Given the immense burden of mental illness, there is dire need for methods that can detect psychological symptoms and enhance current treatments at a large scale. Due to the central role of language in psychotherapy, we tested whether a linguistic marker of psychological distance could be used as an indicator of a client's symptom severity throughout treatment. Using a large sample of psychotherapy transcripts, we found replicable evidence that linguistic distance indeed tracks internalizing symptoms at both within- and between-person levels and that clustering analyses reveal groups of participants who differ in both their symptom severity and treatment gains. These results support a theoretical model in which linguistic distance reflects healthy emotion regulation, making linguistic distance a tool for measuring mental health and treatment progress.

Both initial analyses of an exploratory dataset and preregistered analyses of a hold-out validation dataset provided consistent evidence that linguistic distance increased over time and tracked internalizing symptoms. Although prior research has shown that using language to distance oneself from aversive stimuli is related to effective emotion regulation (29-33), here we demonstrate the translational impact of this basic finding by showing its relationship with psychological symptoms in a naturalistic and longitudinal dataset of psychotherapy transcripts. 
Not only does this result support a theoretical model in which distancing language facilitates healthy emotion regulation, it also lends evidence to theories that emphasize the transdiagnostic role of emotion regulation in psychopathology $(20,45,46)$. Additionally, the discovery of a stable small relation to psychopathology in a large dataset clarifies mixed results obtained from prior studies, most of which used small samples (41-44). Furthermore, this is the first study to show that the relationship between linguistic distance and internalizing symptoms exists at both between-person and within-person levels. This extends prior research that focused only on between-level relationships $(34,47)$, providing strong evidence that linguistic distance can serve as a diagnostic and prognostic indicator of symptom severity, even as symptom levels fluctuate over the course of treatment.

However, evidence for mediation (i.e., that increasing linguistic distance explained reduced symptoms across treatment) was inconsistent across exploratory and validation datasets. These inconsistencies suggest that linguistic distance either plays a very small mechanistic role in psychotherapy or that it merely reflects rather than reduces internalizing symptoms in therapy. Although this result runs contrary to hypotheses, it prompts future research that can decipher how linguistic distance tracks internalizing symptoms without serving a mediating role. Examining relations between language and symptoms at closer timescales or examining measures of a client's actual emotion regulation or psychological distancing skills (rather than their linguistic correlates) could provide better tests of this underlying model.

Finally, clustering approaches provided replicable evidence that trajectories of linguistic distance can predict treatment outcomes and symptom severity. In particular, we found that both starting levels and slopes of linguistic distance related to symptom severity and treatment response, respectively. These results supplement the regression results described above to 
demonstrate that client language is intimately linked to mental health and treatment response, allowing us to deduce clinically relevant diagnostic and prognostic information from linguistic data alone. These clustering methods pave the way for more sophisticated machine-learning approaches that could provide accurate clinical predictions from a client's linguistic data. Additionally, differences between the temporal and social distancing metrics presented in the supplement carry several interesting implications for psychotherapy. These analyses showed that temporal distance clusters differed significantly in their treatment outcomes but not baseline symptoms, but social distance clusters differed in internalizing symptom severity but not changes in symptoms over time. Consequently, linguistic measures of social distance (i.e., pronoun use) may provide a trait-like measure of overall internalizing dysfunction, whereas temporal distance may reflect within-person shifts in one's retreating symptoms. Future research that parses temporal and social distance at both the linguistic and phenomenological levels (i.e., assessing client's experienced tendency to dilate their psychological focus away from themselves and/or the present moment) could shed further light on these hallmark symptoms of depression and anxiety, as well as the role of this process in successful treatment (see Supplementary Information for further discussion).

A strength of the current study is its unprecedented scale and naturalism, made possible through an inclusive approach to analyses. Indeed, using unfiltered data reduced experimenter degrees of freedom and provided the most conservative test of our research questions. However, taking such an unconstrained approach means that substantial noise remains in the data. The decisions to (i) include every text message (even if they are extremely short or may not be related to therapeutic interventions; e.g., messages about scheduling), (ii) include all participants (even those who provided very few text messages), and (iii) averaging linguistic data over a 3 
week period to match the frequency of symptom measures could add noise and cloud accurate assessments of effect sizes. Developing principled inclusion criteria and filtering methods could improve assessments of effect sizes of relationships between variables. Nonetheless, this study serves as a foundational litmus test of these relationships and future studies using machine learning and natural language processing approaches could further refine effect size estimates.

Potentially because of this naturalistic approach, effect sizes for linguistic relationships were consistently small. This indicates that we observed subtle linguistic shifts over treatment and that linguistic interventions may only provide a small "nudge" when it comes to actual clinical impact. However, it is important to remember that linguistic distance was a byproduct, not a target of treatment, meaning that observing this effect in the context of an inclusive and naturalistic dataset provides strong support for the underlying theoretical model. Additionally, it's possible that the higher level of noise at the text level and relatively small number of withinperson symptom measurements (i.e., 3-5) compared to the high number of subjects (i.e., thousands) could have rendered within-subjects relations much weaker than between-person relations. Future research should use principles noted above to reduce noise and improve estimates of effect sizes, examine whether there are moderators that shift "for whom" these effects work, and increase the frequency of within-person symptom sampling to test whether within-person effects are actually larger than those estimated here. That said, there are reasons to value these small effects. Researchers have recently argued that celebrating small effects is key to developing a replicable psychological science (48), and even small effect sizes can have a large impact when they are employed on large scale. For example, if $25 \%$ of 327 million Americans suffer from psychopathology in any given year, helping patients recover just 1 day 
faster will restore 82 million days of human productivity. As such, it would also be prudent to conduct cost-benefit analyses to quantify the actual impact of these interventions.

The current findings advance the field's ability to detect mental health problems from language alone. To work towards deployable tools with real-world impact, future research should address a few key limitations of the current study. First, we propose a theoretical model in which linguistic distance reflects emotion regulation abilities, which increase across time and ultimately improve internalizing symptoms. However, the current study does not include measures of emotion regulation, leaving it unclear what linguistic distance represents in this study. It could indeed reflect improved emotion regulation, but it could also reflect myriad other constructs (e.g., avoidance, improved therapeutic alliance). Future studies that empirically evaluate how adaptive emotion regulation fits in the proposed model are needed. Second, because there was no control group in this study, we cannot infer that Talkspace conversations were causally involved in either clients' decreased symptoms across time or their increased linguistic distance across time. Studies that utilize active control conditions are needed to determine causal relationships. These experiments could also (i) give a clearer sense of the downstream impacts of these relationships (e.g., if they can causally reduce symptoms in the long term) and (ii) adjudicate between the direction of language-symptom relationships. The current study tests one direction (i.e., that language predicts and explains symptom changes), and although this association emerges, mediations were inconsistent. Follow-up experiments that include measures at a fine timescale could compare this direction with its reverse (i.e., that symptoms predict and explain language changes).

Third, although we demonstrate an overall relationship between higher linguistic distance and reduced symptoms, it is possible that distancing is not always an adaptive strategy. Indeed, 
substantial data show that "experiential avoidance" (i.e., pushing away internal or external stressors; ostensibly increasing distance) is maladaptive, whereas mindfully attending to the present moment (ostensibly decreasing distance) is adaptive (49-52). Similarly, depression and anxiety are stereotypically seen as disorders in which people are overly focused on past losses or future threats, respectively. Why then would distancing from the present be helpful if habitually being "away from" the present moment is associated with psychopathology? One way to reconcile this apparent paradox is to consider that people suffering from depression and anxiety might not be distancing themselves from the present moment to think about the past and future; instead they are pulling these past and future moments into the present, seeing them with very low psychological distance, and acting as if they are currently happening (e.g., "I can't believe I am such a failure" or "catastrophe is imminent"). As such, it is possible that learning to resist the avoidant strategies of worry and rumination requires taking a distanced perspective on that maladaptive habit and gaining skills to interrupt these processes. Mindful awareness is one strategy to do just that, as even though it requires attending to the present moment, it also calls for viewing one's thoughts as detached and separate from oneself $(51,53)$, a highly distanced perspective. These are initial attempts at resolving the puzzle of how a distanced perspective may facilitate psychological health, even though prior research establishes avoidance as unhelpful and present-focused mindful awareness as helpful. However, these possibilities require additional empirical investigation, a line of research that would benefit from incorporating emerging frameworks that emphasize the contextual nature of emotion regulation to parse when and in which contexts high distance is adaptive (54-56).

In conclusion, this study used a large dataset of therapeutic exchanges to show that the psychological distance encoded in one's speech reflects one's level of internalizing symptoms 
and can even track within-person changes in symptom severity across time in treatment. Although mixed results emerged for whether linguistic distance played a mediating role in treatment outcomes, the current study lends support to the theoretical model suggesting that linguistic distancing tracks both emotion regulation and mental health. Findings extend prior research, foster new research questions, and lay the foundation for future tools that can use linguistic tools to both detect individuals suffering from psychopathology and guide interventions that reduce human suffering.

\section{Methods}

\section{Participants}

This study included data from a random sample of 6,229 clients who utilized the digital psychotherapy service Talkspace (https://www.talkspace.com/) between 2016 and 2019. Given the longitudinal focus of this study, participants were only included if they had completed at least three symptom inventory questionnaires spanning at least six weeks of treatment. For included clients, we downloaded (i) a fully deidentified record of all text message exchanges between the client and their Talkspace therapist, (ii) their responses to measures of depression and anxiety, and (iii) their self-reported demographics. Talkspace clients and therapists agreed to third-parties conducting research on their data as a part of the terms of use (https://www.talkspace.com/public/terms). The Harvard University IRB designated the current study not human research (IRB18-1583) as the study utilized pre-existing de-identified data for which consent to research was provided. The overall sample of 6,229 participants was randomly divided into an exploratory dataset $\left(N_{\mathrm{e}}=3,729 ; 60 \%\right)$ and a validation dataset $\left(N_{\mathrm{v}}=2,500 ; 40 \%\right)$, with analyses of the validation dataset only occurring after preregistering analyses and hypotheses (see https://osf.io/r5gn2). Participant demographics are displayed in Table 1. 


\section{Therapy Platform}

Talkspace is a digital mental health platform that provides session-based teletherapy, as well as asynchronous messaging therapy, from which these data were drawn. Potential clients register with the service and begin by describing their presenting complaint and treatment goals with a consultation therapist. This information enables the system to provide the client with three licensed National Committee for Quality Assurance (NCQA) credentialed therapist options. These recommendations are based on each therapist's history with demographically and diagnostically similar clients. The chosen "primary therapist" then treats the client. Clients can purchase live phone and video sessions, but most clients select the messaging-only plan (98.1\% in this dataset, Table 1).

Clients may send therapists messages whenever they wish using the HIPAA-compliant smartphone-based application or the Talkspace website. Therapists respond by messages during designated hours. Clients have the option to transfer to a different primary therapist, but most clients interacted with only one therapist (i.e., $82.5 \%$ in the full dataset, Table 1). Mean length of text messages in the full dataset was approximately 80 words, providing substantial data for linguistic analysis (Table 1).

\section{Symptom Assessments}

Procedure. Symptom questionnaires were sent to clients via the messaging platform approximately every 3 weeks over the course of therapy. The link to complete questionnaires expired only when the next set of questionnaires were sent (i.e., participants could complete questionnaires whenever they would like after receiving the link, up until the next questionnaire administration). The date on which participants completed the questionnaire was recorded. This date was transformed into a measure of their current time in therapy at that symptom 
measurement by computing the number of days between questionnaire completion and the start of therapy (i.e., the date of the first text message between the client and their primary therapist).

Depression symptoms. Symptoms of depression were measured using the eight-item Personal Health Questionnaire (PHQ-8), a validated and widely used tool for assessing depressive symptoms (57). Participants rated how often over the last two weeks they had been bothered by eight of the nine symptoms of major depressive disorder (i.e., anhedonia, low mood, sleep disturbance, fatigue, appetite disturbance, low self-esteem, concentration difficulties, and psychomotor agitation or slowing). Responses were made on a four-point scale $(0=$ not at all, $1=$ several days, $2=$ more than half the days, $3=$ nearly every day). Responses were summed to provide a measure of overall depression symptom severity, with scores ranging from 0 to 24 . Unlike the PHQ-9, the PHQ-8 does not include an item assessing suicidal ideation. However, studies have shown that the PHQ-8 and PHQ-9 provide equivalently sensitive and valid measures of depressive symptoms (58-60).

Anxiety symptoms. Anxiety symptoms were assessed using the seven-item Generalized Anxiety Disorder Questionnaire (GAD-7) (61), a widely-used and validated measure of anxiety symptoms. Participants rated how often over the last two weeks they had been bothered by core symptoms of generalized anxiety disorder (i.e., feelings of anxiety, uncontrollable worrying, difficulty relaxing, restlessness, irritability, and fears of catastrophic outcomes). Responses were made on a four-point scale $(0=$ not at all, $1=$ several days, $2=$ more than half the days, $3=$ nearly every day) and summed to provide a measure of overall anxiety symptom severity, with scores ranging from 0 to 21 . 


\section{Data Processing}

Producing a combined measure of internalizing symptoms. Preliminary analyses in the exploratory dataset revealed that scores on the PHQ-8 and GAD-7 were strongly related to each other (within-person correlation using the stats $B y$ function in the psych package (62): $\left.r_{\mathrm{e}}=.70, p_{\mathrm{e}}<.001, r_{\mathrm{v}}=.69, p_{\mathrm{v}}<.001\right)$. We consequently collapsed these two measures into a single assessment of internalizing symptoms by summing the two scales together, as has been done in prior work (63). Nonetheless, preregistered supplementary analyses were conducted on depression and anxiety scores separately both to present these individual statistics and to show that results were largely equivalent across the two measures (Table S2).

Text processing. We developed code in R Version 4.0.4 (64) to extract individual text messages from Talkspace text records. Text messages were extracted with their corresponding date and time of delivery, as well as the author of the text (i.e., client or therapist). Linguistic distance for each text was first computed following prior work $(29,30,65)$. However, analyses

of the exploratory dataset suggested that overall pronoun and verb use increased over the course of treatment (presumably due to changes in topics of conversation). These overall shifts across time made this measure unsuited to the current study (see Supplementary Information). We consequently developed more precise measures of linguistic distance that controlled for overall shifts in verb and pronoun use over the course of treatment. Linguistic Inquiry and Word Count (LIWC) (66) software was used to compute the percentage of words that were verbs (divided into past, preset, and future tense) and pronouns (divided into first-person singular, first-person plural, second-person, third-person singular, and third-person plural). We computed a temporal distance score for each text message by computing the proportion of verbs that were not in the present-tense [i.e., (past + future) / (past + future + present)]. Similarly, we computed a social 
distance score for each text message by computing the proportion of pronouns that were not first-person singular [i.e., (second-person + first-person plural + third-person singular + thirdperson plural) / (second-person + first-person plural + third-person singular + third-person plural + first-person singular)]. Temporal distance scores were treated as missing for text messages that included no verbs $(7.1 \%$ of client text messages for the exploratory and $6.9 \%$ of validation dataset), and social distance scores were treated as missing for text messages that included no pronouns $(9.4 \%$ of client text messages for exploratory and $9.1 \%$ of validation dataset).

We then averaged these two measures at the text level into a single combined linguistic distance score $(11.2 \%$ messages were unusable due to no pronouns or verbs used in exploratory dataset and $10.9 \%$ in validation dataset). This revised measure of linguistic distance (i) captures the relative focus on temporal and social targets that are distanced from the present moment, and (ii) accounts for overall differences in verb and pronoun use across treatment. Analyses of social and temporal distance as separate metrics are presented in the Supplementary Information.

Aligning text and questionnaire data. Text data were collected at a more granular timescale (i.e., minutes, hours, days) compared to symptom measures (i.e., every 3 weeks). We computed the mean linguistic distance in users' text messages within the $\sim 3$ week periods between symptom assessments and aligned these averages with the symptom assessments completed at the end of each of these observation periods. We quantified time (i.e., days in therapy) by computing the number of days between the date questionnaires were completed and the beginning of therapy (i.e., the date of the first text message between the client and the primary therapist). This resulted in a dataset comprised of baseline symptom measures (at time $=0$ ), symptom measures at each subsequent symptom measurement point, and the mean 
linguistic distance of client text messages sent before each of these symptom measurements, all nested within participants.

Consideration of exclusion criteria. It is worth noting that we adopted an inclusive approach to analyzing this real-world dataset. Although criteria could have been developed to exclude participants (e.g., minimum number of text messages, minimum initial symptom severity, type of subscription) or text messages (e.g., minimum word count), we refrained from imposing experimenter-defined cutoffs as much as possible. Given the novelty of this naturalistic analysis, we chose to take an inclusive approach to provide unbiased insight into research questions, but the presence of unfiltered noise should be noted when interpreting results.

\section{Research Questions, Analyses, and Hypotheses}

Are internalizing symptoms, linguistic distance, and time in treatment related? We

first tested the "arms" of a mediation model in which linguistic distance mediates reductions in internalizing symptoms over time in treatment. This involved using mixed-effect models test for linear relationships between (i) days in treatment and internalizing symptoms, (ii) days in treatment and linguistic distance and (iii), linguistic distance and internalizing symptoms. We hypothesized (i) that time in therapy would be negatively related to symptoms, (ii) linguistic distance would be positively related to time in therapy, and (iii) that linguistic distance would be negatively related to internalizing symptoms at both within-person and between-person levels of analysis.

For this third relationship, it was important to decompose measures of linguistic distance into within-person and between-person components within this longitudinal design $(67,68)$. This is because a relationship between linguistic distance and internalizing symptoms could emerge in mixed-effect models either (i) because as people increase their linguistic distance, their 
symptoms reduce (a within-person relationship) or (ii) because individuals who overall have higher linguistic distance have lower symptoms than individuals who overall have lower linguistic distance (a between-person relationship). We consequently followed prior work in decomposing linguistic distance into within-person and between-person components and used these components in mixed-effects regressions (67-72). A variable representing the withinperson fluctuation in linguistic distance was created by subtracting each individual's mean linguistic distance score from the score of each of their observations, producing a variable representing within-person deviation, centered around their individual mean. Then, a variable representing between-person variance in linguistic distance was constructed by subtracting the overall group mean of linguistic distance from that participant's average temporal distance. This produced a variable that was constant for each participant and represented how their mean level deviated from the group's mean. These within-person and between-person variables were then entered simultaneously in mixed-effects models testing relations between linguistic distance and internalizing symptoms.

Does linguistic distance mediate symptom reduction? We next conducted mediation analyses to formally test whether increasing linguistic distance over the course of treatment mediated symptom changes, using measures of linguistic distance that had been decomposed into their within-person and between-person components. Typically, mediation analyses utilize bootstrapping methods (i.e., randomly sampling from the original dataset with preplacement thousands of times) to generate many samples from which a confidence window can be constructed to test the significance of mediation model (73). However, the appropriate method for bootstrapping multilevel data is not clear, as random samples can be drawn at the participant level, at the observation level, or at both participant and observation levels. We thus used 
Bayesian analytic procedures — which do not involve bootstrapping methods — for our mediation analyses to sidestep this issue (70).

Like the mixed-effects models described above, Bayesian regression models included a random effect of participant to account for the multi-level nature of the dataset. To provide relatively unbiased starting points for Bayesian analyses, we supplied weakly informative priors (gaussian distribution of $M=0, S D=10$ ) for all regressors in the models. Bayesian analyses were implemented using the Stan language in R (74). Two Markov chains used the Monte Carlo No U-Turn Sampler (75) to approximate the posterior distribution of each regressor across 12,500 iterations, with the first 2,500 iterations discarded as burn-in. The indirect effect (i.e., the $a \times b$ pathway for the within-person parameter) and proportion mediated (i.e., indirect effect / [indirect effect + direct effect $] \times 100)$ were computed for each mediation model. A significant mediation was determined when the $95 \%$ credible range (CR) of posterior density for the indirect effect did not include 0 . We hypothesized that within-person increases in linguistic distance would mediate decreased symptoms across therapy.

Can symptoms be inferred from linguistic patterns alone? Finally, we used clustering approaches to supplement the regression models utilized above. One limitation of regressions is the extent of aggregation that is required to align text and questionnaire data, resulting in loss of information and introduction of noise. As such, we utilized finite mixture regression techniques (76), which analyze data at the text level. In essence, mixture regressions identify clusters of individuals depending on similarities of joint distributions among variables. This means that participants who tend to have the same relationship between two variables are grouped together. In this case, we used mixture regressions to cluster individuals based on how their linguistic distance in individual text messages varied across time (e.g., grouping clients whose linguistic 
distance increased over time into one cluster and grouping clients whose linguistic distance decreased over time into a different cluster). This allowed us to test whether text data could be used to draw inferences about clients' symptoms and treatment outcomes.

Mixture regression models were conducted on a dataset that included the linguistic distance score for every text message that clients sent to their primary therapist over the course of therapy. For added precision, time in therapy was quantified as a decimal value that included the proportion of a day that had passed since the first text sent between the client and the therapist. We conducted mixture regression analyses that grouped participants into one, two, three, and four clusters, and then selected the number of clusters that provided the best fit, as determined by AIC. For additional stability and model fit, mixture regressions for each cluster size were implemented 10 times (to account for subtle differences that can emerge depending on random starting points of the clustering algorithm), and the best fitting model was selected. Mixture regressions included a random effect of subject to account for nesting of text messages within subjects. We then conducted analyses in the aggregated dataset described above (i.e., in which linguistic data were averaged to match the timeline of symptom inventories) to test how each measure of linguistic distance varied across time in each cluster (using mixed-effects models), as well as how clusters differed in baseline and final internalizing symptoms (using ANOVAs and post-hoc Tukey tests), and how they differed in their change in internalizing symptom scores (i.e., analyzing final - baseline internalizing symptoms change scores using ANCOVAs to control for baseline symptom levels; $[77,78])$.

Additional preregistered analyses. All analyses were initially only conducted in the exploratory dataset of 3,720 participants and analyses of the 2,500 participants in the validation dataset occurred following preregistration. Note that we preregistered analyzing social and 
temporal components of linguistic distancing measure separately and in the revision process, we decided to combine these into a single measure. Results and conclusions are largely the same when each component is analyzed, and all preregistered analyses are provided in the

Supplementary Information, including separate analyses of depression and anxiety symptoms. We also preregistered an additional set of analyses related to the role of therapist linguistic distancing in treatment outcomes. Because the current paper focuses on client language, we have reserved analyses of therapist language for a subsequent report focused on interpersonal processes in therapy.

Model building. Mixed-effects models all included a random intercept for subject. We followed conventional model building steps to test whether adding random slopes improved model fit (as determined by a lower AIC and a significant model comparison). For both the exploratory and validation datasets, these steps consistently revealed that adding a random slope for time in therapy significantly improved model fit. Hence, a random slope of time in therapy was added to all models that included this variable as a fixed effect. Random slopes for models without time as a predictor (e.g., relating client temporal distance and internalizing symptoms) were included when doing so improved model fit. Linear mixed-effect model regression estimates are reported in standardized units (i.e., $\beta$ ). Note that there are several methods for computing standardized $\beta$ s in mixed-effects models, and here coefficients are standardized at their relevant "level" (i.e., in relation to within-person or between-person variance) using the "pseudo" option of the "standardize_parameters" function in the effectsize package (79). We characterize effect sizes according to conventions for correlation coefficients (i.e., $\sim .1=$ small, $\sim .3=$ medium, $\sim .5=$ large) (80). To provide an additional estimate of effect sizes in mixed-effects models, we report the proportion variance explained by each predictor (i.e., semi-partial $R_{\beta}^{2}$ ) 
following the conventions described by Edwards et al. $(81,82)$ and using Satterthwaite estimation of degrees of freedom. Regression estimates for Bayesian mediation models are reported in their raw unstandardized form (i.e., $b$ ), but we report the proportion mediated as the key effect size for each mediation model. We use eta squared (i.e., $\eta^{2}$ ) to report the effect size of one-way ANOVAs and partial eta squared (i.e., $\eta_{\mathrm{p}}{ }^{2}$ ) for ANCOVAs that control for baseline symptoms.

Software. LIWC 2007 (66) was used to extract word class frequencies from text messages. Mixed-effects models were conducted in lme4 (83), with p-values calculated using the lmerTest package (84). Standardized betas of linear mixed-effects models were extracted using the effectsize package (79). Bayesian analyses were conducted using the brms package $(85,86)$. Mixture regressions were conducted in the flexmix package (76).

Data availability. Talkspace data can be made available upon completion of a Data Use Agreement and data security review with Talkspace. Markdowns documenting analytic code and results of all analyses are available at https://osf.io/u98r3/. 


\section{Acknowledgements}

Thanks to James Gross, Ethan Kross, and the Cambridge Writing Group for helpful comments.

Thanks to the Harvard University's Institute for Quantitative Social Science for guidance on statistical analyses. This work was supported by a National Science Foundation Graduate Research Fellowship to ECN (DGE1144152). 


\section{REFERENCES}

1. Global Burden of Disease Collaborative Network (2017) Global Burden of Disease Study 2016 (GBD 2016) results.

2. Kessler RC, et al. (2009) The global burden of mental disorders: An update from the WHO World Mental Health (WMH) surveys. Epidemiol Psichiatr Soc 18(1):23-33.

3. Merwin E, Hinton I, Dembling B, Stern S (2003) Shortages of rural mental health professionals. Arch Psychiatr Nurs 17(1):42-51.

4. Andrews G, Sanderson K, Slade T, Issakidis C (2000) Why does the burden of disease persist? Relating the burden of anxiety and depression to effectiveness of treatment. Bull World Health Organ 78(4):446-454.

5. Lancet Global Mental Health Group (2007) Scale up services for mental disorders: A call for action. Lancet 370(9594):1241-1252.

6. Kazdin AE, Rabbitt SM (2013) Novel models for delivering mental health services and reducing the burdens of mental illness. Clin Psychol Sci 1(2):170-191.

7. Kazdin AE, Blase SL (2011) Rebooting psychotherapy research and practice to reduce the burden of mental illness. Perspect Psychol Sci 6(1):21-37.

8. Perle JG, Nierenberg B (2013) How psychological telehealth can alleviate society's mental health Burden: A literature review. J Technol Hum Serv 31(1):22-41.

9. Holmes EA, et al. (2018) The Lancet Psychiatry Commission on psychological treatments research in tomorrow's science. The Lancet Psychiatry 5(3):237-286.

10. Rathbone AL, Prescott J (2017) The use of mobile apps and SMS messaging as physical and mental health interventions: Systematic review. J Med Internet Res 19(8):1-13.

11. Hull TD, Mahan K (2017) A study of asynchronous mobile-enabled SMS text 
psychotherapy. Telemed e-Health 23(3):240-247.

12. DellaCrosse M, Mahan K, Hull TD (2019) The effect of messaging therapy for depression and anxiety on employee productivity. J Technol Behav Sci 4(1):1-5.

13. Andrews G, Cuijpers P, Craske MG, McEvoy P, Titov N (2010) Computer therapy for the anxiety and depressive disorders is effective, acceptable and practical health care: A metaanalysis. PLoS One 5(10):e13196.

14. Hull TD, Malgaroli M, Connolly P, Feuerstein S, Simon NM (2020) The treatment effectiveness of asynchronous text therapy for depression and anxiety: A naturalistic observation study. BMC Psychiatry.

15. Brans K, Van Mechelen I, Rimé B, Verduyn P (2014) To share, or not to share? Examining the emotional consequences of social sharing in the case of anger and sadness. Emotion 14(6):1062-1071.

16. Rimé B (2009) Emotion elicits the social sharing of emotion: Theory and empirical review. Emot Rev 1(1):60-85.

17. Rimé B, Bouchat P, Paquot L, Giglio L (2020) Intrapersonal, interpersonal, and social outcomes of the social sharing of emotion. Curr Opin Psychol 31:127-134.

18. Gross JJ (1998) The emerging field of emotion regulation: An integrative review. Rev Gen Psychol 2(5):271-99.

19. Gross JJ (2015) Emotion regulation: Current status and future prospects. Psychol Inq 26(1):1-26.

20. Aldao A, Nolen-Hoeksema S, Schweizer S (2010) Emotion-regulation strategies across psychopathology: A meta-analytic review. Clin Psychol Rev 30(2):217-237.

21. Fernandez KC, Jazaieri H, Gross JJ (2016) Emotion regulation: A transdiagnostic 
perspective on a new RDoC domain. Cognit Ther Res 40(3):426-440.

22. Gross JJ, Jazaieri H (2014) Emotion, emotion regulation, and psychopathology: An affective science perspective. Clin Psychol Sci 2(4):387-401.

23. Radkovsky A, McArdle JJ, Bockting CLH, Berking M (2014) Successful emotion regulation skills application predicts subsequent reduction of symptom severity during treatment of major depressive disorder. J Consult Clin Psychol 82(2):248-62.

24. Berking M, et al. (2008) Emotion-regulation skills as a treatment target in psychotherapy. Behav Res Ther 46(11):1230-1237.

25. Berking M, Ebert D, Cuijpers P, Hofmann SG (2013) Emotion regulation skills training enhances the efficacy of inpatient cognitive behavioral therapy for major depressive disorder: A randomized controlled trial. Psychother Psychosom 82(4):234-245.

26. Daros AR, et al. (2021) A meta-analysis of emotional regulation outcomes in psychological interventions for youth with depression and anxiety. Nat Hum Behav 5(10):1443-1457.

27. Parkinson C, Liu S, Wheatley T (2014) A common cortical metric for spatial, temporal, and social distance. $J$ Neurosci 34(5):1979-87.

28. Trope Y, Liberman N (2010) Construal-level theory of psychological distance. Psychol Rev 117(2):440-463.

29. Nook EC, Schleider JL, Somerville LH (2017) A linguistic signature of psychological distancing in emotion regulation. $J$ Exp Psychol Gen 146(3):337-346.

30. Nook EC, Vidal Bustamante CM, Cho HY, Somerville LH (2020) Use of linguistic distancing and cognitive reappraisal strategies during emotion regulation in children, adolescents, and young adults. Emotion 20(4):525-540. 
31. Kross E, et al. (2017) Third-person self-talk reduces ebola worry and risk perception by enhancing rational thinking. Appl Psychol Heal Well-Being 9(3):387-409.

32. Kross E, et al. (2014) Self-talk as a regulatory mechanism: How you do it matters. J Pers Soc Psychol 106(2):304-324.

33. Orvell A, et al. (2021) Does distanced self-talk facilitate emotion regulation across a range of emotionally intense experiences? Clin Psychol Sci 9(1):68-78.

34. Tackman AM, et al. (2019) Depression, negative emotionality, and self-referential language: A multi-lab, multi-measure, and multi-language-task research synthesis. J Pers Soc Psychol 116(5):817-834.

35. Edwards ER, Wupperman P (2017) Emotion regulation mediates effects of alexithymia and emotion differentiation on impulsive aggressive behavior. Deviant Behav 38(10):1160-1171.

36. Brockmeyer T, et al. (2015) Me, myself, and I: self-referent word use as an indicator of self-focused attention in relation to depression and anxiety. Front Psychol 6(1564). doi:10.3389/fpsyg.2015.01564.

37. Todorov G, Mayilvahanan K, Cain C, Cunha C (2020) Context- and subgroup-specific language changes in individuals who develop PTSD after trauma. Front Psychol 11(May):1-11.

38. Zimmermann J, Brockmeyer T, Hunn M, Schauenburg H, Wolf M (2017) First-person pronoun use in spoken language as a predictor of future depressive symptoms: Preliminary evidence from a clinical sample of depressed patients. Clin Psychol Psychother 24(2):384-391.

39. Stirman SW, Pennebaker JW (2001) Word use in the poetry of suicidal and nonsuicidal 
poets. Psychosom Med 63:517-522.

40. Berry-Blunt AK, Holtzman NS, Donnellan MB, Mehl MR (2021) The story of "I" tracking: Psychological implications of self-referential language use. Soc Personal Psychol Compass (September):1-14.

41. Althoff T, Clark K, Leskovec J (2016) Large-scale analysis of counseling conversations: An application of natural language processing to mental health. Trans Assoc Comput Linguist 4:463-476.

42. Haug S, Strauss B, Gallas C, Kordy H (2008) New prospects for process research in group therapy: Text-based process variables in psychotherapeutic Internet chat groups. Psychother Res 18(1):88-96.

43. Van Staden CW, Fulford KWMM (2004) Changes in semantic uses of first person pronouns as possible linguistic markers of recovery in psychotherapy. Aust $N Z J$ Psychiatry 38(4):226-232.

44. Arntz A, Hawke LD, Bamelis L, Spinhoven P, Molendijk ML (2012) Changes in natural language use as an indicator of psychotherapeutic change in personality disorders. Behav Res Ther 50(3):191-202.

45. Barlow DH, et al. (2011) The Unified Protocol for Transdiagnostic Treatment of Emotional Disorders: Therapist Guide (Oxford University Press, New York).

46. Mennin DS (2004) Emotion regulation therapy for generalized anxiety disorder. Clin Psychol Psychother 11(1):17-29.

47. Edwards T, Holtzman NS (2017) A meta-analysis of correlations between depression and first person singular pronoun use. $J$ Res Pers 68:63-68.

48. Götz FM, Gosling SD, Rentfrow PJ (2021) Small effects: The indispensable foundation 
for a cumulative psychological science. Perspect Psychol Sci. doi:10.1177/1745691620984483.

49. Kashdan TB, Barrios V, Forsyth JP, Steger MF (2006) Experiential avoidance as a generalized psychological vulnerability: Comparisons with coping and emotion regulation strategies. Behav Res Ther 44(9):1301-1320.

50. Kashdan TB, et al. (2014) A contextual approach to experiential avoidance and social anxiety: Evidence from an experimental interaction and daily interactions of people with social anxiety disorder. Emotion. doi:10.1037/a0035935.

51. Wielgosz J, Goldberg SB, Kral TRA, Dunne JD, Davidson RJ (2019) Mindfulness meditation and psychopathology. Annu Rev Clin Psychol 15:285-316.

52. Kabat-Zinn J (2003) Mindfulness-based interventions in context: Past, present, and future. Clin Psychol Sci Pract 10(2):144-156.

53. Hayes SC, Wilson KG (2003) Mindfulness: Method and process. Clin Psychol Sci Pract 10(2):161-165.

54. Aldao A (2013) The future of emotion regulation research: Capturing context. Perspect Psychol Sci 8(2):155-172.

55. Ford BQ, Troy AS (2019) Reappraisal Reconsidered: A Closer Look at the Costs of an Acclaimed Emotion-Regulation Strategy. Curr Dir Psychol Sci 28(2):195-203.

56. Satpute AB, Nook EC, Cakar M (2020) The role of language in the construction of emotion and memory. Neuroscience of Enduring Change: Implications for Psychotherapy, eds Lane R, Nadel L (Oxford University Press, New York, NY).

57. Kroenke K, et al. (2009) The PHQ-8 as a measure of current depression in the general population. J Affect Disord 114(1-3):163-173. 
58. Razykov I, Ziegelstein RC, Whooley MA, Thombs BD (2012) The PHQ-9 versus the PHQ-8 - Is item 9 useful for assessing suicide risk in coronary artery disease patients ? Data from the Heart and Soul Study. J Psychosom Res 73(3):163-168.

59. Corson K, Gerrity M, Dobscha S (2004) Screening for depression and suicidality in a VA primary care setting: 2 items are better than 1 item. Am J Manag Care 10(11 Pt 2):839845.

60. Shin C, Lee SH, Han KM, Yoon HK, Han C (2019) Comparison of the usefulness of the PHQ-8 and PHQ-9 for screening for major depressive disorder: Analysis of psychiatric outpatient data. Psychiatry Investig 16(4):300-305.

61. Spitzer R, Kroenke K, Williams J, Lowe B (2006) A brief measure for assessing generalized anxiety disorder. Arch Intern Med 166:1092-7.

62. Revelle W (2016) psych: Procedures for personality and psychological research (Northwestern University, Evanston, IL).

63. Kroenke K, et al. (2016) Patient health questionnaire anxiety and depression scale: Initial validation in three clinical trials. Psychosom Med 78(6):716-727.

64. R Core Team (2021) R: A language and environment for statistical computing.

65. Mehl MR, Robbins ML, Holleran SE (2012) How taking a word for a word can be problematic: Context-dependent linguistic markers of extraversion and neuroticism. $J$ Methods Meas Soc Sci 3(2):30-50.

66. Pennebaker JW, Booth RJ, Francis ME (2007) Linguistic Inquiry and Word Count: LIWC [Computer software].

67. Enders CK, Tofighi D (2007) Centering predictor variables in cross-sectional multilevel models: A new look at an old issue. Psychol Methods 12(2):121-138. 
68. Bolger N, Laurenceau J-P (2013) Intensive Longitudinal Methods: An Introduction to Diary and Experience Sampling Research (Guilford, New York, NY).

69. Nook EC, Flournoy JC, Rodman AM, Mair P, McLaughlin KA (2021) Emotion differentiation buffers the impact of stressful events on internalizing symptoms in adolescence. Clin Psychol Sci. doi:10.31234/osf.io/q4uy8.

70. Rodman AM, et al. (2020) A year in the social life of a teenager: Within-person fluctuations in stress, phone communication, and anxiety and depression. Under Rev.

71. Starr LR, Hershenberg R, Li YI, Shaw ZA (2017) When feelings lack precision: Low positive and negative emotion differentiation and depressive symptoms in daily life. Clin Psychol Sci 5(4):613-631.

72. Vidal Bustamante CM, et al. (2020) Within-person fluctuations in stressful life events, sleep, and anxiety and depression symptoms during adolescence: A multi-wave prospective study. J Child Psychol Psychiatry.

73. Davison AC, Hinkley D V. (1997) Bootstrap Methods and Their Applications (Cambridge University Press, Cambridge).

74. Stan Development Team (2017) Stan modeling language: User's guide and reference manual. Available at: http://mc-stan.org/manual.html.

75. Hoffman MD, Gelman A (2014) The no-U-turn sampler: Adaptively setting path lengths in Hamiltonian Monte Carlo. J Mach Learn Res 15:1593-1623.

76. Leisch F (2004) FlexMix: A general framework for finite mixture models and latent class regression in R. J Stat Softw 11(8):1-18.

77. Vickers AJ, Altman DG (2001) Analysing controlled trials with baseline and follow up measurements. Br Med J 323(7321):1123-1124. 
78. Clifton L, Clifton DA (2019) The correlation between baseline score and post-intervention score, and its implications for statistical analysis. Trials 20(1):4-9.

79. Ben-Shachar M, Lüdecke D, Makowski D (2020) effectsize: Estimation of effect size indices and standardized parameters. J Open Source Softw 5(56):2815.

80. Cohen J (1992) A power primer. Psychol Bull 112(1):155-159.

81. Edwards LJ, Muller KE, Wolfinger RD, Qaqish BF, Schabenberger O (2008) An R2 statistic for fixed effects in the linear mixed model. Stat Med 27(29):6137-6157.

82. Jaeger BC, Edwards LJ, Das K, Sen PK (2017) An R2 statistic for fixed effects in the generalized linear mixed model. J Appl Stat 44(6):1086-1105.

83. Bates D, Mächler M, Bolker BM, Walker SC (2015) Fitting linear mixed-effects models using lme4. J Stat Softw 67(1):1-48.

84. Kuznetsova A, Brockhoff PB, Christensen RHB (2017) lmerTest package: Tests in linear mixed effects models. J Stat Softw 82(13). doi:10.18637/jss.v082.i13.

85. Bürkner PC (2017) brms: An R package for Bayesian multilevel models using Stan. J Stat Softw 80(1). doi:10.18637/jss.v080.i01.

86. Bürkner PC (2018) Advanced Bayesian multilevel modeling with the R package brms. $R J$ 10(1):395-411. 


\section{TABLES}

Table 1. Sample and platform description.

\begin{tabular}{|c|c|c|c|}
\hline & Full sample & Exploratory subsample & Validation subsample \\
\hline \multicolumn{4}{|l|}{ Gender } \\
\hline Female & $4,742(77.4 \%)$ & $2,857(77.7 \%)$ & $1,885(77.0 \%)$ \\
\hline Male & $1,306(21.3 \%)$ & $766(20.8 \%)$ & $540(22.1 \%)$ \\
\hline Transgender female & $11(0.2 \%)$ & $8(0.2 \%)$ & $3(0.1 \%)$ \\
\hline Transgender male & $11(0.2 \%)$ & $5(0.1 \%)$ & $6(0.2 \%)$ \\
\hline Gender queer & $27(0.4 \%)$ & $21(0.6 \%)$ & $6(0.2 \%)$ \\
\hline Gender variant & $6(0.1 \%)$ & $3(0.1 \%)$ & $3(0.1 \%)$ \\
\hline Other & $20(0.3 \%)$ & $16(0.4 \%)$ & $4(0.2 \%)$ \\
\hline No response & 106 & 53 & 53 \\
\hline \multicolumn{4}{|l|}{$\operatorname{Age}^{\mathrm{a}}$} \\
\hline $18-25$ & $1,031(22.2 \%)$ & $606(21.8 \%)$ & $425(22.8 \%)$ \\
\hline $26-35$ & $2,536(54.6 \%)$ & $1,517(54.6 \%)$ & $1,019(54.8 \%)$ \\
\hline $36-49$ & $871(18.8 \%)$ & $526(18.9 \%)$ & $345(18.5 \%)$ \\
\hline $50+$ & $203(4.4 \%)$ & $131(4.7 \%)$ & $72(3.9 \%)$ \\
\hline No response & 1,588 & 949 & 639 \\
\hline \multicolumn{4}{|l|}{ Race } \\
\hline Caucasian & $1,172(60.4 \%)$ & $698(60.3 \%)$ & $474(60.5 \%)$ \\
\hline African American & $284(14.6 \%)$ & $179(15.5 \%)$ & $105(13.4 \%)$ \\
\hline Asian & $140(7.2 \%)$ & $82(7.1 \%)$ & $58(7.4 \%)$ \\
\hline Hispanic & $120(6.2 \%)$ & $66(5.7 \%)$ & $54(6.9 \%)$ \\
\hline Native American & $5(0.3 \%)$ & $2(0.2 \%)$ & $3(0.4 \%)$ \\
\hline Other & $195(10.1 \%)$ & $115(9.9 \%)$ & $80(10.2 \%)$ \\
\hline Declined to identify & $24(1.2 \%)$ & $15(1.3 \%)$ & $9(1.1 \%)$ \\
\hline No response & 4,289 & 2,572 & 1,717 \\
\hline \multicolumn{4}{|l|}{ Education level } \\
\hline Less than high school & $28(0.5 \%)$ & $14(0.4 \%)$ & $14(0.7 \%)$ \\
\hline High school & $808(15.7 \%)$ & $477(15.3 \%)$ & $331(16.2 \%)$ \\
\hline Associate's degree & $78(1.5 \%)$ & $40(1.3 \%)$ & $38(1.9 \%)$ \\
\hline Some college no degree & $200(3.9 \%)$ & $126(4.0 \%)$ & $74(3.6 \%)$ \\
\hline Bachelor's degree & $3,683(71.4 \%)$ & $2,238(71.9 \%)$ & $1,445(70.7 \%)$ \\
\hline Master's degree & $260(5.0 \%)$ & $163(5.2 \%)$ & $97(4.7 \%)$ \\
\hline Professional degree & $43(0.8 \%)$ & $23(0.7 \%)$ & $20(1.0 \%)$ \\
\hline Doctoral degree & $56(1.1 \%)$ & $31(1.0 \%)$ & $25(1.2 \%)$ \\
\hline No response & 1,073 & 617 & 456 \\
\hline \multicolumn{4}{|l|}{ Symptom measures } \\
\hline $\begin{array}{l}\text { Baseline internalizing } \\
\text { symptoms }\end{array}$ & $M=22.21, S D=9.90$ & $M_{\mathrm{e}}=21.99, S D_{\mathrm{e}}=9.89$ & $M_{\mathrm{v}}=22.54, S D_{\mathrm{v}}=9.92$ \\
\hline Final internalizing symptoms & $M=15.16, S D=9.87$ & $M_{\mathrm{e}}=14.95, S D_{\mathrm{e}}=9.79$ & $M_{\mathrm{v}}=15.48, S D_{\mathrm{v}}=10.00$ \\
\hline Baseline depression symptoms & $M=11.03, S D=5.82$ & $M_{\mathrm{e}}=10.93, S D_{\mathrm{e}}=5.84$ & $M_{\mathrm{v}}=11.18, S D_{\mathrm{v}}=5.80$ \\
\hline Final depression symptoms & $M=7.56, S D=5.58$ & $M_{\mathrm{e}}=7.44, S D_{\mathrm{e}}=5.54$ & $M_{\mathrm{v}}=7.73, S D_{\mathrm{v}}=5.62$ \\
\hline Baseline anxiety symptoms & $M=11.18, S D=5.04$ & $M_{\mathrm{e}}=11.06, S D_{\mathrm{e}}=5.02$ & $M_{\mathrm{v}}=11.36, S D_{\mathrm{v}}=5.06$ \\
\hline Final anxiety symptoms & $M=7.60, S D=4.90$ & $M_{\mathrm{e}}=7.50, S D_{\mathrm{e}}=4.83$ & $M_{\mathrm{v}}=7.75, S D_{\mathrm{v}}=4.99$ \\
\hline \multicolumn{4}{|l|}{ Therapy and text qualities } \\
\hline Text-only subscription & $6,108(98.1 \%)$ & $3,659(98.1 \%)$ & $2,449(98.0 \%)$ \\
\hline
\end{tabular}




\begin{tabular}{lccc} 
Number of client messages & 759,706 & 455,379 & 304,327 \\
Length of client messages & $M=80.81$, & $M_{\mathrm{e}}=81.61$, & $M_{\mathrm{v}}=79.61$, \\
(words) & $\mathrm{S} D=146.80$ & $S D_{\mathrm{e}}=147.02$ & $S D_{\mathrm{v}}=146.46$ \\
Number of therapist messages & 461,911 & 273,208 & 188,703 \\
Length of therapist messages & $M=82.69$, & $M_{\mathrm{e}}=84.32$, & $M_{\mathrm{v}}=80.33$, \\
(words) & $S D=107.30$ & $S D_{\mathrm{e}}=108.00$ & $S D_{\mathrm{v}}=106.23$ \\
Present-tense verbs per & $M=8.73$, & $M_{\mathrm{e}}=8.81$, & $M_{\mathrm{v}}=8.61$, \\
message & $S D=15.10$ & $S D_{\mathrm{e}}=15.11$ & $S D_{\mathrm{v}}=15.09$ \\
Past-tense verbs per message & $M=3.87$, & $M_{\mathrm{e}}=3.89$, & $M_{\mathrm{v}}=3.83$, \\
& $S D=9.34$ & $S D_{\mathrm{e}}=9.34$ & $S D_{\mathrm{v}}=9.34$ \\
Future-tense verbs per & $M=0.82$, & $M_{\mathrm{e}}=0.83$, & $M_{\mathrm{v}}=0.81$, \\
message & $S D=1.80$ & $S D_{\mathrm{e}}=1.81$ & $S D_{\mathrm{v}}=1.79$ \\
First-person singular pronouns & $M=8.13$, & $M_{\mathrm{e}}=8.17$, & $M_{\mathrm{v}}=8.06$, \\
per message & $S D=14.50$ & $S D_{\mathrm{e}}=14.49$ & $S D_{\mathrm{v}}=14.51$ \\
Other pronouns per message & $M=3.75$, & $M_{\mathrm{e}}=3.78$, & $M_{\mathrm{v}}=3.71$, \\
& $S D=9.38$ & $S D_{\mathrm{e}}=9.38$ & $S D_{\mathrm{v}}=9.38$ \\
Number of therapists & $M=1.21, S D=0.51$ & $M_{\mathrm{e}}=1.21, S D_{\mathrm{e}}=0.51$ & $M_{\mathrm{v}}=1.22, S D_{\mathrm{v}}=0.52$ \\
Number of symptom measures & $M=3.45, S D=0.51$ & $M_{\mathrm{e}}=3.45, S D_{\mathrm{e}}=0.51$ & $M_{\mathrm{v}}=3.45, S D_{\mathrm{v}}=0.51$ \\
\multicolumn{1}{c}{ Exactly 3 measures } & $3,453(55 \%)$ & $2,058(55 \%)$ & $1,395(56 \%)$ \\
\multicolumn{1}{c}{ Exactly 4 measures } & $2,741(44 \%)$ & $1,651(44 \%)$ & $1,090(44 \%)$ \\
Exactly 5 measures & $35(1 \%)$ & $20(1 \%)$ & $15(1 \%)$ \\
Days between start of therapy & $M=63.93, S D=11.90$ & $M_{\mathrm{e}}=63.94, S D_{\mathrm{e}}=11.89$ & $M_{\mathrm{v}}=63.91, S D_{\mathrm{v}}=11.92$ \\
and final symptom measure & & &
\end{tabular}

Notes: Percentages ignore clients who did not respond to each demographic question. ${ }^{\text {a}}$ Participants reported their age by selecting from the pre-defined bins presented above. 


\section{FIGURES}

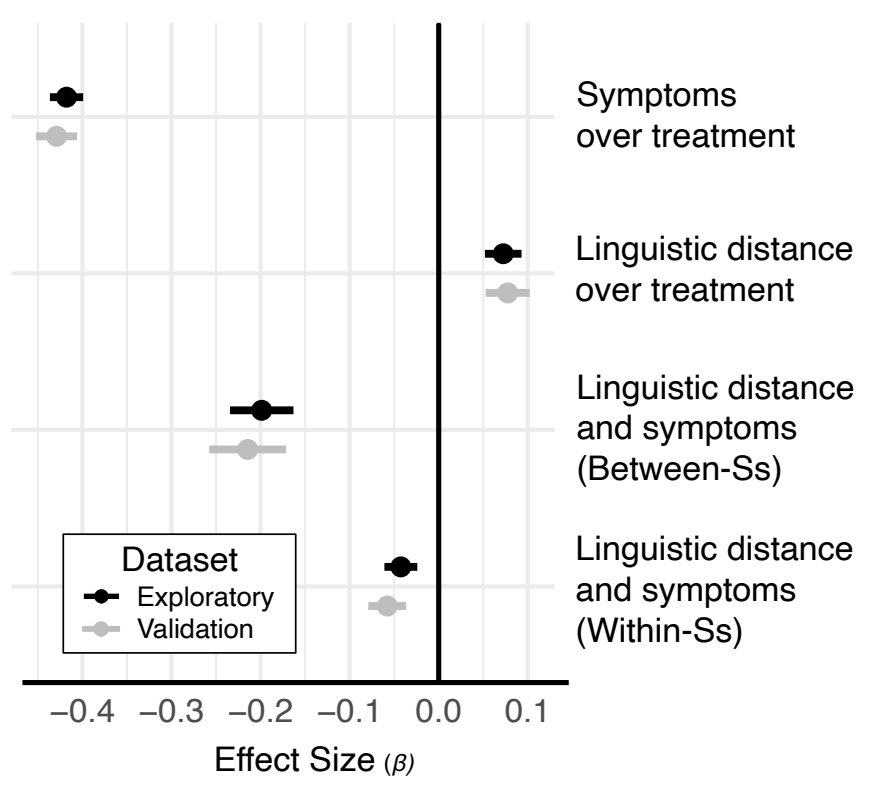

Figure 1. Effect size plot for mixed-effects regressions depicting relations between internalizing symptoms, linguistic distance, and time in treatment within the exploratory (black) and validation (grey) datasets. All 95\% confidence intervals do not include 0 , indicating significant associations. 


\section{A Exploratory Dataset}

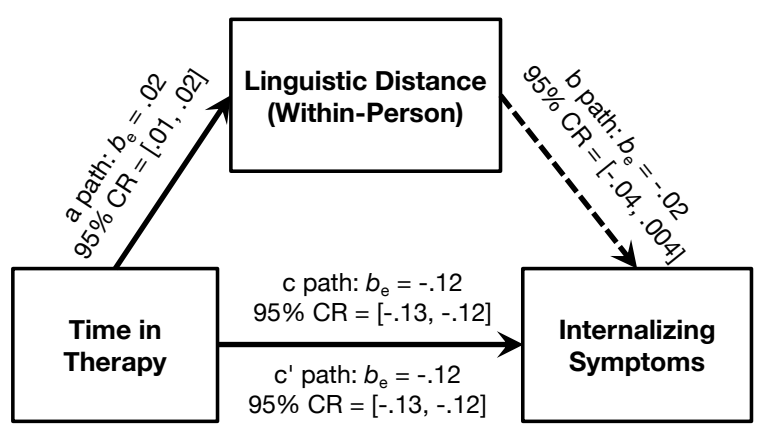

Non-Significant Indirect Effect $b_{\mathrm{e}}=-.003,95 \% \mathrm{CR}=[-.0007, .0001]$ Proportion mediated $=0.3 \%$
B Validation Dataset

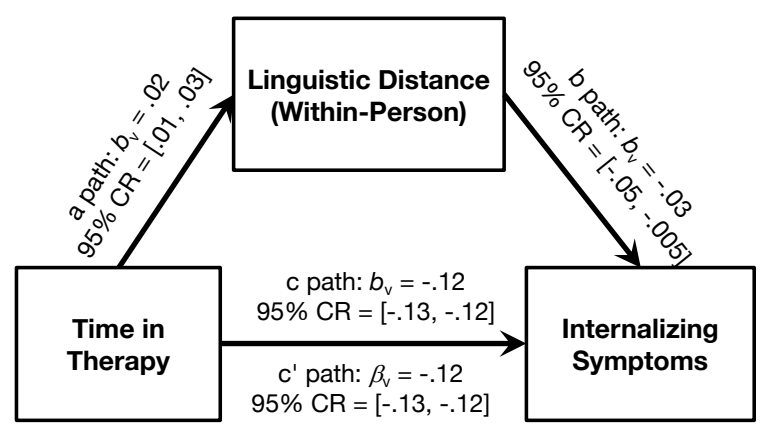

Significant Indirect Effect

$b_{v}=-.0006,95 \% \mathrm{CR}=[-.001,-.00008]$

Proportion mediated $=0.5 \%$

Figure 2. Bayesian mediation models testing whether within-person variance in client linguistic distance mediated changes in internalizing symptoms across time in the (A) exploratory and (B) validation datasets. The $95 \%$ credible range (CR) for the indirect effect included 0 for the exploratory but not validation dataset, providing mixed evidence for the proposed mediation model. Median regression estimates are reported from Bayesian regression models, with their corresponding 95\% CRs. 


\section{Exploratory Dataset}
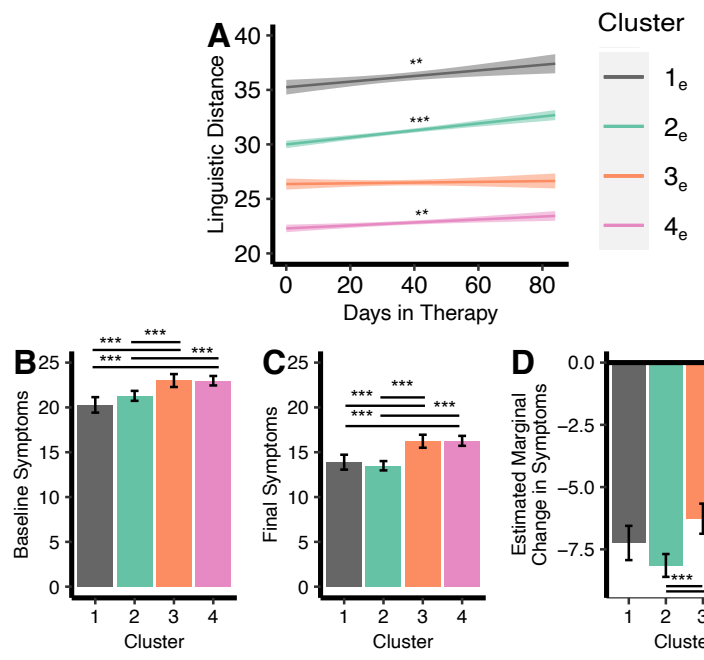
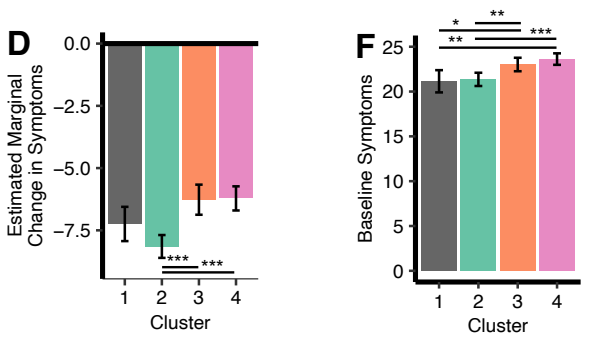

Validation Dataset
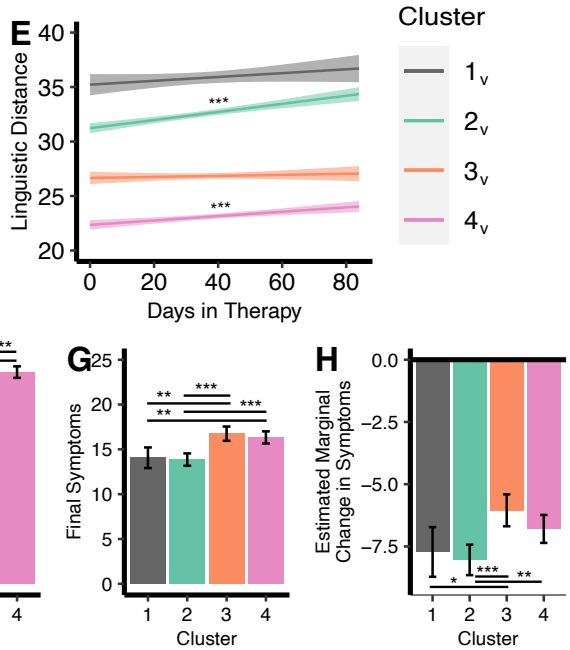

Figure 3. Results of the finite mixture regression clustering participants based on the linguistic distance in their texts over the course of therapy for the exploratory (A-D) and validation (E-G) datasets. (A \& E) Four clusters were identified, which differed in starting and ending linguistic distance as well as the slope of changes in linguistic distance over the course of treatment. (B \& F) Clusters differed significantly in baseline internalizing symptoms such that the clusters that started with higher linguistic distance (i.e., clusters 1 and 2) had lower symptoms at the start of treatment than those that started with lower linguistic distance (i.e., clusters 3 and 4). (C \& G) Clusters differed significantly in final internalizing symptoms, with clusters 1 and 2 also reporting significantly fewer symptoms than clusters 3 and 4. (D \& H) Estimated marginal means of changes in internalizing symptoms across clusters (accounting for baseline symptom levels). Cluster 2, which had both a high starting level of linguistic distance and the strongest increase over time, achieved the best treatment response, significantly stronger than clusters 3 and $4 .{ }^{* * *} p<.001, * * p<.01, * p<.05$. 


\title{
Supplementary Information for
}

\section{Linguistic measures of psychological distance track symptom levels and treatment outcomes in a large set of psychotherapy transcripts}

\author{
Erik C. Nook, Thomas D. Hull, Matthew K. Nock, \& Leah H. Somerville \\ Corresponding Author: Erik C. Nook \\ Email: enook@princeton.edu
}

This PDF file includes:

Supplementary Text 1: Testing the traditional linguistic distance measure Supplementary Text 2: Discussion of supplementary analyses in Tables S1 and S2 Table S1. Analyses of temporal and social distance subcomponents Table S2. Analyses of depression and anxiety symptoms

Figure S1. Results of the temporal distance mixture regression in the exploratory dataset Figure S2. Results of the temporal distance mixture regression in the validation dataset Figure S3. Results of the social distance mixture regression in the exploratory dataset Figure S4. Results of the social distance mixture regression in the validation dataset SI References 


\section{Supplementary Text 1: Testing the Traditional Linguistic Distance Measure}

We initially quantified linguistic distance using a traditional approach, following prior research associating this metric with psychological distance and emotion regulation (1-3). Linguistic Inquiry and Word Count (LIWC; Pennebaker, Booth, \& Francis, 2007) software was used to compute the percentage of words that fall into each component of the linguistic distance composite (i.e., first-person singular pronouns, present-tense verbs, discrepancy words, articles, and words longer than 6 letters). These percentages were z-scored across participants, and use of first-person singular pronouns, present-tense verbs, and discrepancy words were reverse-scored by multiplying z-scores by -1 . Resulting scores were averaged to produce linguistic distance scores for each text message. However, the behavior of this measure was confusing: It was negatively related to internalizing symptoms, $\beta_{\mathrm{e}}=-.06, p_{\mathrm{e}}<.001, R^{2} \beta_{\mathrm{e}}=.02$, but it decreased over time in treatment, $\beta_{\mathrm{e}}=-.05, p_{\mathrm{e}}<.001, R^{2} \beta_{\mathrm{e}}=.01$. We conducted additional analyses in the exploratory dataset to elucidate why this measure suggested that higher distancing was related to better mental health but also decreased over time, as mental health improved.

Because prior studies using this approach did not have a longitudinal structure, we sought to verify that this approach indeed captured how distanced individuals were within a longitudinal design. The traditional measure of linguistic distance described above presupposes that having a larger proportion of words in a passage that refer to "close" psychological targets (i.e., present-tense verbs and first-person singular pronouns) signifies lower distance. In longitudinal designs, it is possible that changes in use close words across time do not necessarily imply opposite changes in distanced words across time. If so, one cannot infer shifts in psychological distance from use of close words alone. Instead, it may be necessary to compute relative measures of linguistic distance, in which use of close words (i.e., the number of present-tense verbs and first-person singular pronouns in a text message) are standardized relative to their relevant word classes (i.e., the overall number of verbs and pronouns, respectively).

Consequently, we investigated how subcomponents of the traditional linguistic distance measure varied across time in clients' text messages in the exploratory dataset. We observed that the percentage of words in clients' text messages that were both present-tense verbs, $\beta_{\mathrm{e}}=.14, p_{\mathrm{e}}<.001, R^{2}{ }_{\mathrm{ee}}=.06$, and first-person singular pronouns, $\beta_{\mathrm{e}}=.06, p_{\mathrm{e}}<.001, R^{2}{ }_{\mathrm{e}}=.01$, increased across time in therapy. Because these word types connote psychological "closeness," this resulted in initial analyses showing that the traditional linguistic distance measure decreased over therapy (see above), contrary to hypotheses. However, when we examined words that connote psychological distance, we found that clients' use of past-tense verbs, $\beta_{\mathrm{e}}=.13, p_{\mathrm{e}}<.001, R^{2} \beta_{\mathrm{e}}=.05$, and future-tense verbs, $\beta_{\mathrm{e}}=.05, p_{\mathrm{e}}<.001, R^{2} \beta_{\mathrm{e}}=.01$, also increased across therapy, as did use of pronouns that were not first-person singular (i.e., words like "you," "we," "she," "he," and "they"), $\beta_{\mathrm{e}}=.05, p_{\mathrm{e}}<.001, R^{2} \beta_{\mathrm{e}}=.01$. These baseline shift in clients' use of verbs and pronouns across time in therapy raised theoretical concerns with using the traditional measure of linguistic distance: Even though clients were using more "close" words at the end of treatment, it is problematic to conclude that they were less distanced if they also used more "distanced" words. Instead, relative measures of linguistic distance that controlled for baseline shifts in verb and pronoun use across treatment were needed.

Following preregistration, we conducted the analyses listed above in the validation dataset, finding consistent results. In the validation dataset, the traditional measure of linguistic distance was negatively related to symptoms, $\beta_{v}=-.07, p_{v}<.001, R^{2}{ }_{s v}=.05$, but it also decreased across time in therapy, $\beta_{v}=-.06, p_{v}<.001, R^{2} \beta_{v}=.01$. Although present-tense verb use rose across time, $\beta_{v}=.11, p_{v}<.001, R^{2} \beta_{v}=03$, so did past-tense verb use, $\beta_{v}=.12, p_{v}<.001, R^{2} \beta_{v}=.04$, and future-tense verb use, $\beta_{v}=.07, p_{v}<.001$, $R^{2} \beta_{v}=.02$. Similarly, although first-person singular pronoun use rose across time in therapy, $\beta_{v}=.07$, $p_{v}<.001, R^{2} \beta_{v}=.01$, so did use of non-first-person singular pronouns, $\beta_{v}=.05, p_{v}<.001, R^{2} \beta_{v}=.01$. As such, we were justified in using the relative measures of temporal and social distance in both the exploratory and validation datasets. We then averaged these together to form a single measure of linguistic distance. Even though we developed this method to account for longitudinal general increases in use of pronouns and verbs, this approach could also be fruitfully applied in cross-sectional studies that don't have a longitudinal design. 


\section{Supplementary Text 2: Discussion of Supplementary Analyses in Tables S1 and S2}

Here, we give a general summary and discussion of the supplementary analyses that focus on the temporal and social subcomponents of linguistic distancing and anxiety/depression symptoms. These results are presented in Tables S1-S2 and Figures S2-S4.

Temporal and social subcomponents. Preregistered analyses used temporal distance (i.e., the proportion of verbs that were not present-tense) and social distance (i.e., the proportion of pronouns that were not first-person singular) as primary dependent variables. In the process of revising the paper, we decided to average together these values into a single measure of linguistic distance. Results are largely identical for all three measures, but we present statistics of these subcomponents in Table S1. Both social and temporal measures increased across time in treatment and were negatively associated with internalizing symptoms at between-person and within-person levels. Notably, the between-person relation between internalizing symptoms and social distance showed the largest linguistic effect ( $\beta s=.19-.21$ ). Mediation models were inconsistent, with only the temporal distance mediation returning a significant indirect effect in the validation dataset. As such, it's possible that shifts in verb use may play a small explanatory role in symptom reduction. Cluster analyses revealed an interesting divergence between temporal and social distance. Clusters based on temporal distance trajectories differed in symptom change scores but not in baseline internalizing symptom levels, whereas clusters based on social distance trajectories differed in baseline and final internalizing symptom levels but not symptom change scores (see Figures S2-S5). These results replicated in the validation dataset.

These subcomponent analyses lead to similar conclusions as results for the combined measure presented in the main text: Linguistic distance rises over therapy, tracks changing symptom levels, and can be used to discover groups of participants who vary in their symptom severity and treatment response. However, there are also some interesting qualitative differences between temporal and social distance that could motivate future research on how these dimensions might relate to symptoms in slightly different ways. In particular, the regression, mediation, and clustering results suggest that temporal distance (i.e., verb use) may more strongly relate to within-person symptom changes, whereas social distance (i.e., pronoun use) may more strongly relate to between-person symptom severity. In other words, social distance may provide a trait-like measure of overall internalizing dysfunction, with more firstperson singular pronoun use reflecting a more static between-person vulnerability to internalizing problems, even if the individual's symptoms are retreating. This aligns with the notion that major depressive disorder is a lifetime diagnosis that merely has phasic episodes of illness (5). As such, pronoun use may reflect the cognitive vulnerabilities that characterize people with major depressive disorder, even when their symptoms are in remission (6-10).

Temporal distance may instead reflect within-person shifts in one's retreating symptoms (e.g., the prevalence of rumination or worry-repetitively thinking about past or future negative experiences; [1113]). Shifts in temporal distance could also reflect other active components of psychological treatment, like meaning making (i.e., being able to create a positive integrated narrative about prior experiences [14]). It is worth noting that in the temporal distance clustering for both exploratory and validation datasets, clusters $A$ and $B$ began with similar levels of linguistic distance but achieved significantly different final internalizing symptom scores, and the same is true for clusters $C$ and $D$. These differences between groups are likely due to their different slopes of linguistic distance over time, further supporting the idea that increasing linguistic distance reflects treatment gains. Future research that parses temporal and social distance at both the linguistic and phenomenological levels (i.e., assessing client's experienced tendency to dilate their psychological focus away from themselves and/or the present moment) could shed further light on these hallmark symptoms of depression and anxiety, as well as the role of this process in successful treatment.

Anxiety and depression symptoms. As can be seen in Table S2, analyses of anxiety and depression scores reveal results that are largely all consistent with what was reported in the main text when the combined internalizing symptom measure was used. Nonetheless, we present statistics from these highly granular analyses for thoroughness in case they are useful for future meta-analyses and to transparently show how summing these scores did or did not affect results. Inconsistencies across measures were constrained to: (i) temporal distance was an inconsistent mediator of depression and anxiety symptoms across the exploratory and validation datasets and (ii) depression scores were significantly different at baseline for temporal distance clusters in the exploratory dataset. 
Table S1. Results of analyses testing relations between temporal and social linguistic distance subcomponents and internalizing symptoms.

Exploratory Dataset Dalidation Dataset

Analysis Statistic Salidatio Significance

Linguistic distance components over time in treatment Temporal distance over time

$\begin{array}{llll}\beta_{\mathrm{e}}=.06 & p_{\mathrm{e}}<.001 & \beta_{\mathrm{v}}=.06 & p_{\mathrm{v}}<.001 \\ \beta_{\mathrm{e}}=.05 & p_{\mathrm{e}}<.001 & \beta_{\mathrm{v}}=.05 & p_{\mathrm{v}}<.001\end{array}$

Social distance over time

$\beta_{\mathrm{e}}=.05$

$p_{\mathrm{e}}<.001$

$\beta_{v}=.05$

$p_{\mathrm{v}}<.001$

Symptoms and linguistic distance Symptoms and raw temporal distance

Symptoms and between-person variance in temporal distance

Symptoms and within-person variance in temporal distance

Symptoms and raw social distance

Symptoms and between-person variance in social distance

Symptoms and within-person variance in social distance

$\begin{array}{llll}\beta_{\mathrm{e}}=-.06 & p_{\mathrm{e}}<.001 & \beta_{\mathrm{v}}=-.08 & p_{\mathrm{v}}<.001 \\ \beta_{\mathrm{e}}=-.10 & p_{\mathrm{e}}<.001 & \beta_{\mathrm{v}}=-.09 & p_{\mathrm{v}}<.001 \\ \beta_{\mathrm{e}}=-.03 & p_{\mathrm{e}}<.001 & \beta_{\mathrm{v}}=-.05 & p_{\mathrm{v}}<.001 \\ \beta_{\mathrm{e}}=-.10 & p_{\mathrm{e}}<.001 & \beta_{\mathrm{v}}=-.12 & p_{\mathrm{v}}<.001 \\ \beta_{\mathrm{e}}=-.19 & p_{\mathrm{e}}<.001 & \beta_{\mathrm{v}}=-.21 & p_{\mathrm{v}}<.001 \\ \beta_{\mathrm{e}}=-.03 & p_{\mathrm{e}}=.001 & \beta_{\mathrm{v}}=-.04 & p_{\mathrm{v}}=.001\end{array}$

Bayesian mediation analyses

c path (Bayesian estimate of symptom change over time)

Indirect effect of within-person temporal distance mediating changes

in symptoms over time

a path for temporal distance mediation

b path for temporal distance mediation

c' path for temporal distance mediation

Indirect effect of within-person social

distance mediating changes in symptoms over time

a path for social distance mediation

b path for social distance mediation

c' path for social distance mediation

$b_{\mathrm{e}}=-.12$
$b_{\mathrm{e}}=-.0003$,
$.2 \%$ mediated
$b_{\mathrm{e}}=.02$
$b_{\mathrm{e}}=-.02$
$b_{\mathrm{e}}=-.12$
$b_{\mathrm{e}}=-.0001$,
$.1 \%$ mediated
$b_{\mathrm{e}}=.02$
$b_{\mathrm{e}}=-.005$
$b_{\mathrm{e}}=-.12$

$[-.13,-.12]$

[-.0006,

$.00005]$

$[.01, .03]$

$[-.03, .002]$

$[-.13,-.12]$

$[-.0004$

$.0001]$

$[.01, .03]$

$[-.02, .01]$

$[-.13,-.12]$
$b_{\mathrm{v}}=-.12$
$b_{\mathrm{v}}=-.0005$
$.4 \%$ mediated
$b_{\mathrm{v}}=.02$
$b_{\mathrm{v}}=-.02$
$b_{\mathrm{v}}=-.12$
$b_{\mathrm{v}}=-.0002$
$.2 \%$ mediated
$b_{\mathrm{v}}=.02$
$b_{\mathrm{v}}=-.01$
$b_{\mathrm{v}}=-.12$

$[-.13,-.12]$

[-.001,

$[.01, .03]$

$[-.04,-.004]$

Finite mixture regressions

Difference in baseline symptoms across temporal distance clusters

Difference in final symptoms across temporal distance clusters

Difference in change in symptoms across temporal distance clusters (controlling for baseline)

Difference in baseline symptoms across social distance clusters

Difference in final symptoms across social distance clusters

Difference in change in symptoms across social distance clusters (controlling for baseline)

$\begin{array}{clcl}F_{\mathrm{e}}=2.59 & p_{\mathrm{e}}=.051 & F_{\mathrm{v}}=2.35 & p_{\mathrm{v}}=.071 \\ F_{\mathrm{e}}=13.10 & p_{\mathrm{e}}<.001 & F_{\mathrm{v}}=14.74 & p_{\mathrm{v}}<.001 \\ F_{\mathrm{e}}=6.90 & p_{\mathrm{e}}<.001 & F_{\mathrm{v}}=9.24 & p_{\mathrm{v}}<.001 \\ & & & \\ F_{\mathrm{e}}=17.03 & p_{\mathrm{e}}<.001 & F_{\mathrm{v}}=11.23 & p_{\mathrm{v}}<.001 \\ F_{\mathrm{e}}=18.82 & p_{\mathrm{e}}<.001 & F_{\mathrm{v}}=10.50 & p_{\mathrm{v}}<.001 \\ F_{\mathrm{e}}=1.54 & p_{\mathrm{e}}=.203 & F_{\mathrm{v}}=0.69 & p_{\mathrm{v}}=.558\end{array}$

$[-.13,-.12]$

$[-.0006$

$.0001]$

$[.01, .03]$

$[-.03, .004]$

$[-.13,-.12]$

Notes: Beta estimates from Bayesian mediation analyses represent the median of posterior estimates of the indirect effect, and values in square brackets represent the $95 \%$ credible range of this estimate. 
Table S2. Results of analyses testing relations between temporal and social linguistic distance components and depression and anxiety symptoms. Depression Symptoms

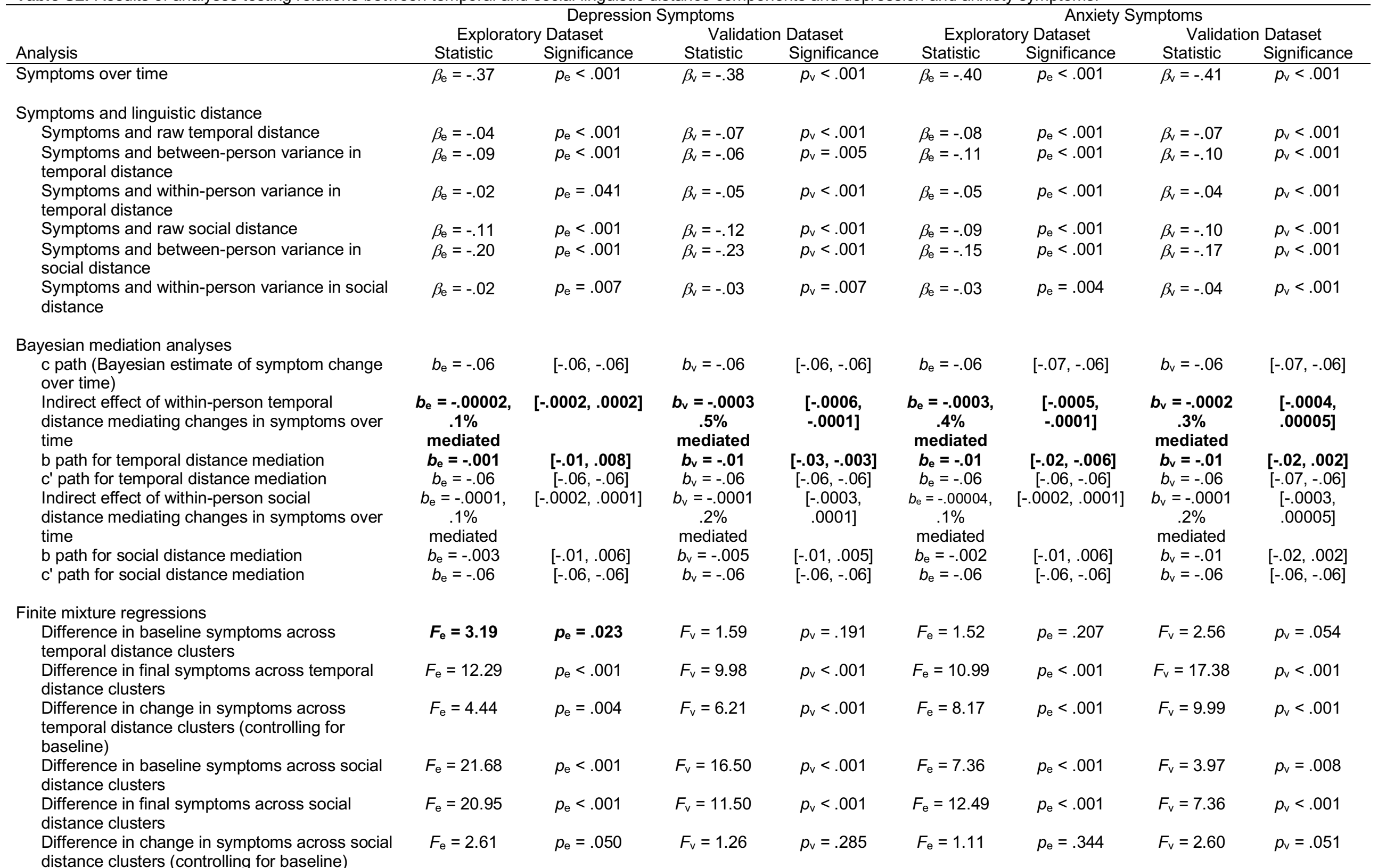

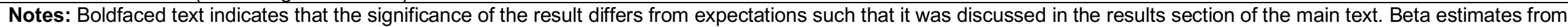

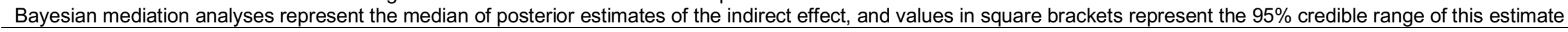



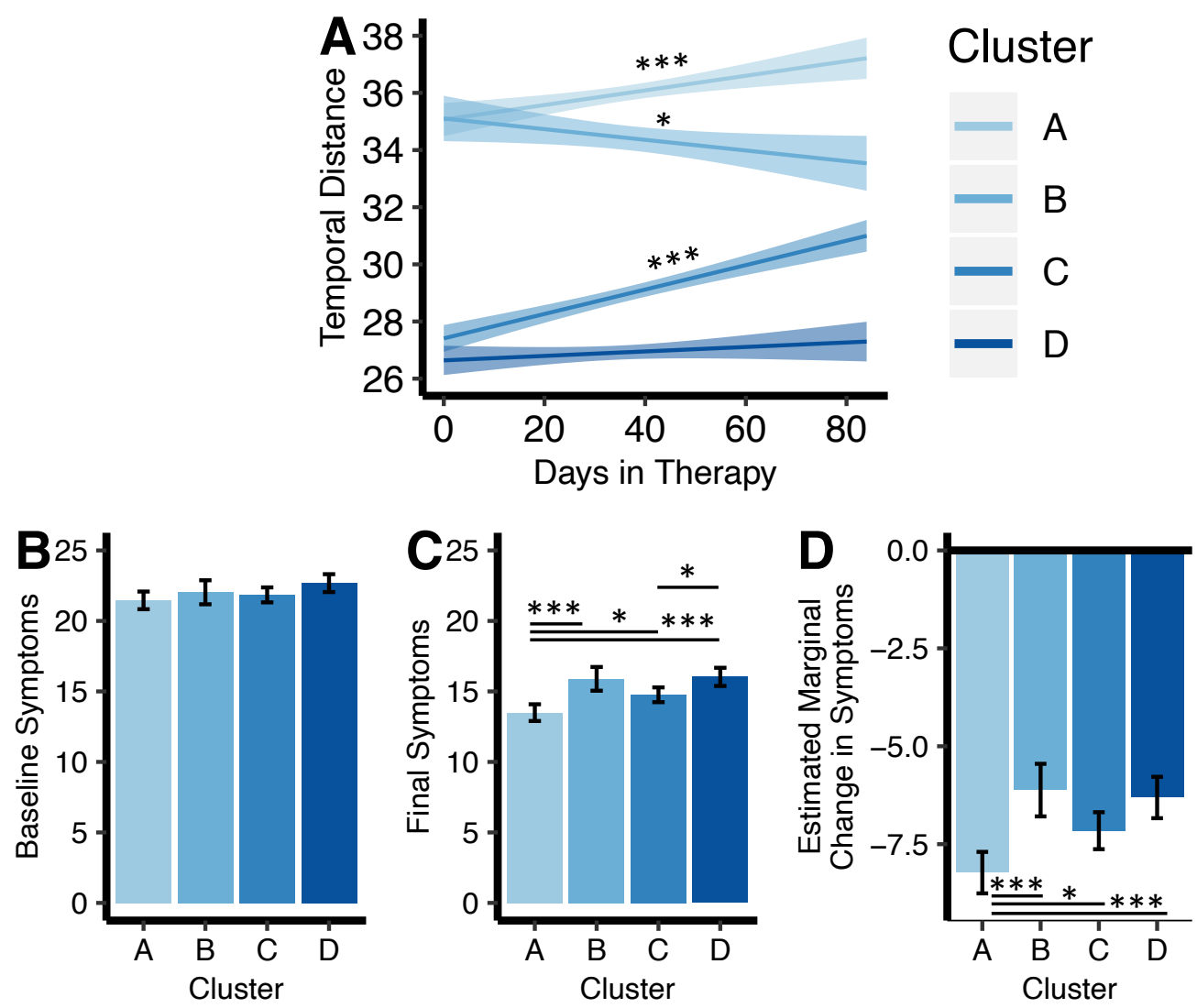

Figure S1. Results of the finite mixture regression clustering participants in the exploratory dataset based on temporal distance. (A) Four clusters were identified. Temporal distance of cluster $A_{e}$ started high and rose over therapy, $\beta_{\mathrm{e}}=.07, p_{\mathrm{e}}<.001, R^{2} \beta_{\mathrm{e}}=.02, N_{\mathrm{e}}=958$, cluster $\mathrm{B}_{\mathrm{e}}$ started high and fell over therapy, $\beta_{\mathrm{e}}=-.05$, $p_{\mathrm{e}}=.045, R^{2} \mathrm{Be}_{\mathrm{e}}=.01, N_{\mathrm{e}}=596$, cluster $C_{\mathrm{e}}$ started low and rose over therapy $\beta_{\mathrm{e}}=.15, p_{\mathrm{e}}<.001, R^{2} \beta_{\mathrm{e}}=.07$, $N_{\mathrm{e}}=1,209$, and cluster $\mathrm{D}_{\mathrm{e}}$ started low and did not significantly change over therapy, $\beta_{\mathrm{e}}=.02, p_{\mathrm{e}}=.241$, $R^{2}{ }_{\mathrm{Be}}=.002, \mathrm{Ne}_{\mathrm{e}}=964$. (B) Clusters did not differ significantly in baseline internalizing symptoms. (C) Clusters differed significantly in final internalizing symptoms, with cluster A reporting significantly fewer symptoms than all other clusters, and cluster $C$ reporting fewer symptoms than cluster D. (D) Clusters differed in their average symptom change over the course of treatment (after controlling for baseline symptom levels), with cluster A experiencing greater reductions than all other clusters. As such, clustering participants based on temporal distance revealed groups of participants who varied in their symptom gains over and above similar baseline symptom levels, with the two clusters that had the strongest increases in temporal distance having lower final symptom scores. ${ }^{* *} p<.001,{ }^{* *} p<.01,{ }^{*} p<.05$. 

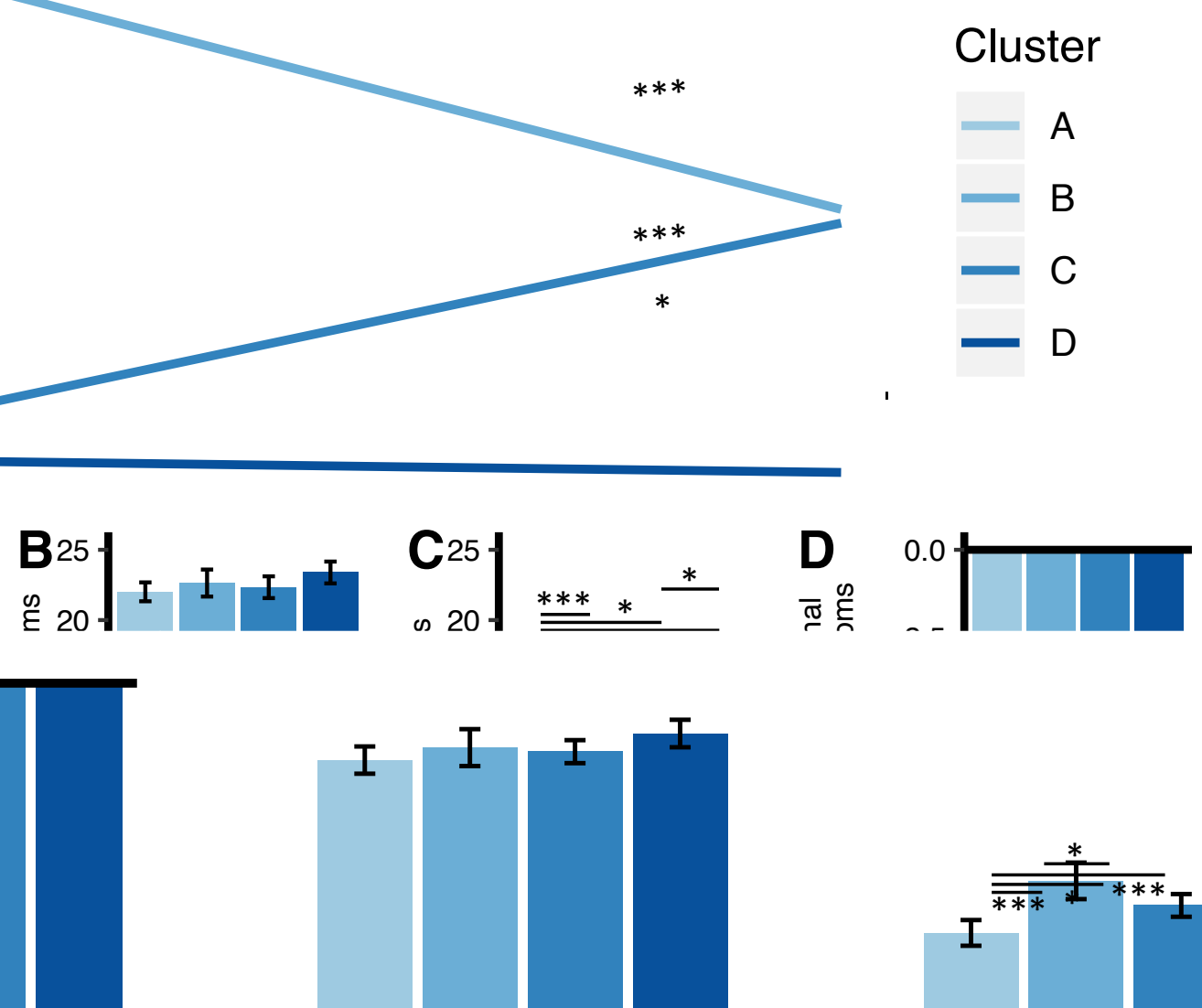

Figure S2. Results of the finite mixture regression clustering participants in the validation dataset based on temporal distance. (A) Four clusters were identified, with temporal distance of cluster $A_{v}$ starting high and increasing over time $\beta_{v}=.07, p_{v}<.001, R_{\beta v}^{2}=.02, N_{v}=787$, cluster $B_{v}$ starting high and not significantly changing over time, $\beta_{\mathrm{v}}=.03, p_{\mathrm{v}}=.264, R^{2} \mathrm{Bv}=.003, N_{\mathrm{v}}=430$, cluster $\mathrm{C}_{\mathrm{v}}$ starting low and increasing over time, $\beta_{v}=.09, p_{v}<.001, R^{2} \beta_{v}=.03, N_{v}=672$, and cluster $D_{v}$ starting low and increasing over time, $\beta_{v}=.05, p_{v}=.034$, $R^{2} \beta_{v}=.008, N_{v}=611$. (B) Clusters did not differ significantly in baseline internalizing symptoms. (C) Clusters differed significantly in final internalizing symptoms, with cluster A reporting significantly fewer symptoms than all other clusters, and cluster $C$ reporting fewer symptoms than cluster D. (D) Clusters differed in their average symptom change over the course of treatment (final - baseline, controlling for baseline), with cluster $\mathrm{A}$ experiencing greater reductions than all other clusters, and cluster $\mathrm{C}$ experiencing greater reductions than cluster $\mathrm{B}$. These results largely replicate those of the exploratory dataset. ${ }^{* * *} p<.001,{ }^{* *} p$ $<.01,{ }^{*} p<.05$. 

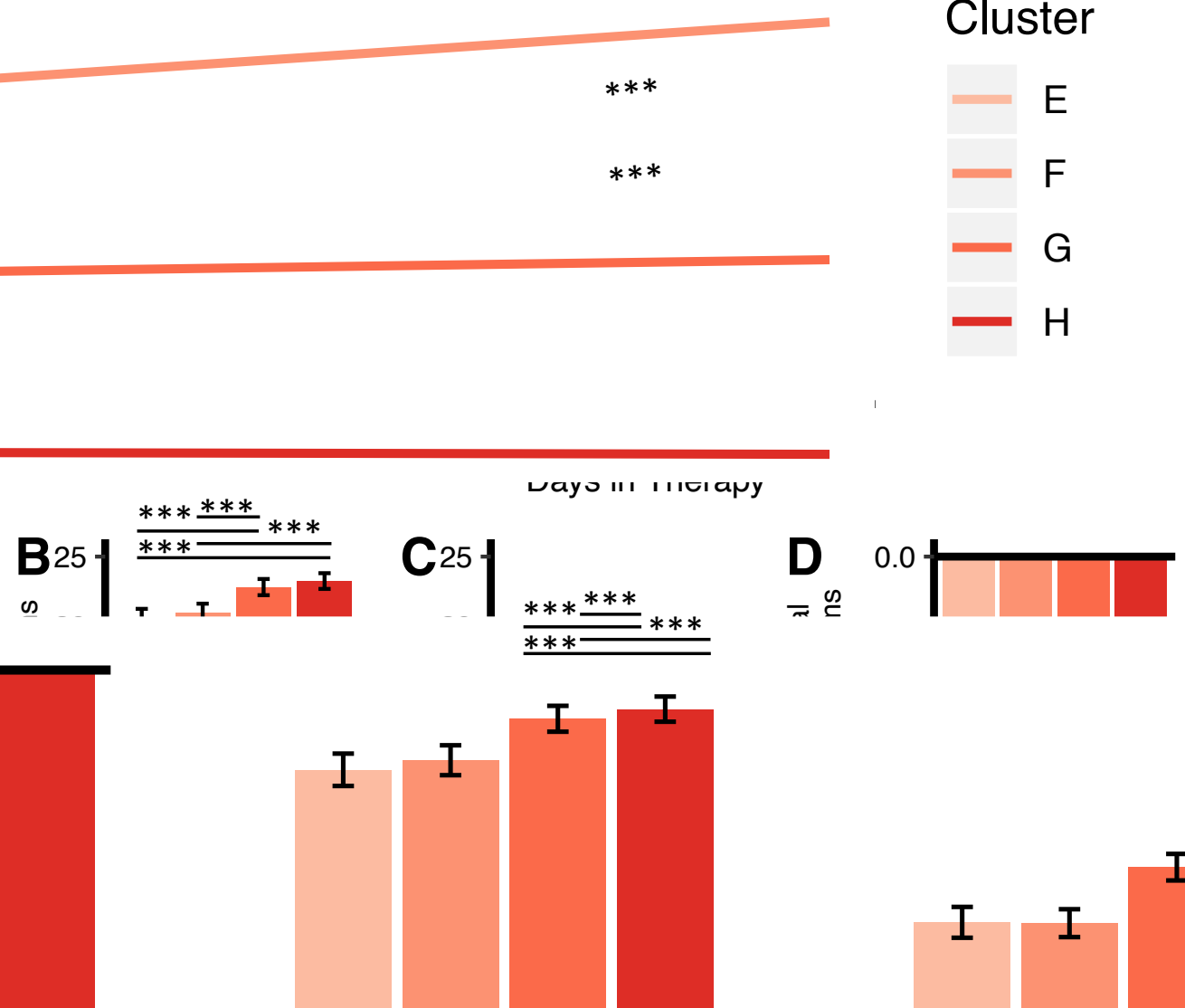

Figure S3. Results of the finite mixture regression clustering participants in the exploratory dataset based on social distance. (A) Four clusters were identified. Social distance for cluster $E_{e}$ started high and increased over therapy, $\beta_{\mathrm{e}}=.13, p_{\mathrm{e}}<.001, R^{2} \beta_{\mathrm{e}}=.05, N_{\mathrm{e}}=775$., cluster $\mathrm{F}_{\mathrm{e}}$ started lower and increased, $\beta_{\mathrm{e}}=.08$, $p_{\mathrm{e}}<.001, R^{2} \mathrm{Be}_{\mathrm{e}}=.02, N_{\mathrm{e}}=902$. cluster $\mathrm{G}_{\mathrm{e}}$ started lower still and did not change significantly over treatment, $\beta_{\mathrm{e}}=.01, p_{\mathrm{e}}=.758, R^{2}{ }_{\mathrm{e}}=.0001, N_{\mathrm{e}}=1,077$, and cluster $\mathrm{H}_{\mathrm{e}}$ started lowest of all and did not change significantly, $\beta_{\mathrm{e}}=-.003, p_{\mathrm{e}}=.898, R^{2} \beta_{\mathrm{e}}=.00002, N_{\mathrm{e}}=973$. (B) Clusters differed significantly in baseline internalizing symptoms, with clusters $E$ and $F$ reporting significantly fewer symptoms than clusters $G$ and $H$. (C) Clusters also differed significantly in final internalizing symptoms, with clusters $E$ and $F$ reporting significantly fewer symptoms than clusters $G$ and $H$. (D) However, clusters did not differ significantly in their average symptom change over the course of therapy (final - baseline, controlling for baseline scores). As such, clustering based on social distance revealed groups of participants that varied tonically in their levels of internalizing symptoms but not their treatment response. ${ }^{* * *} p<.001$. 

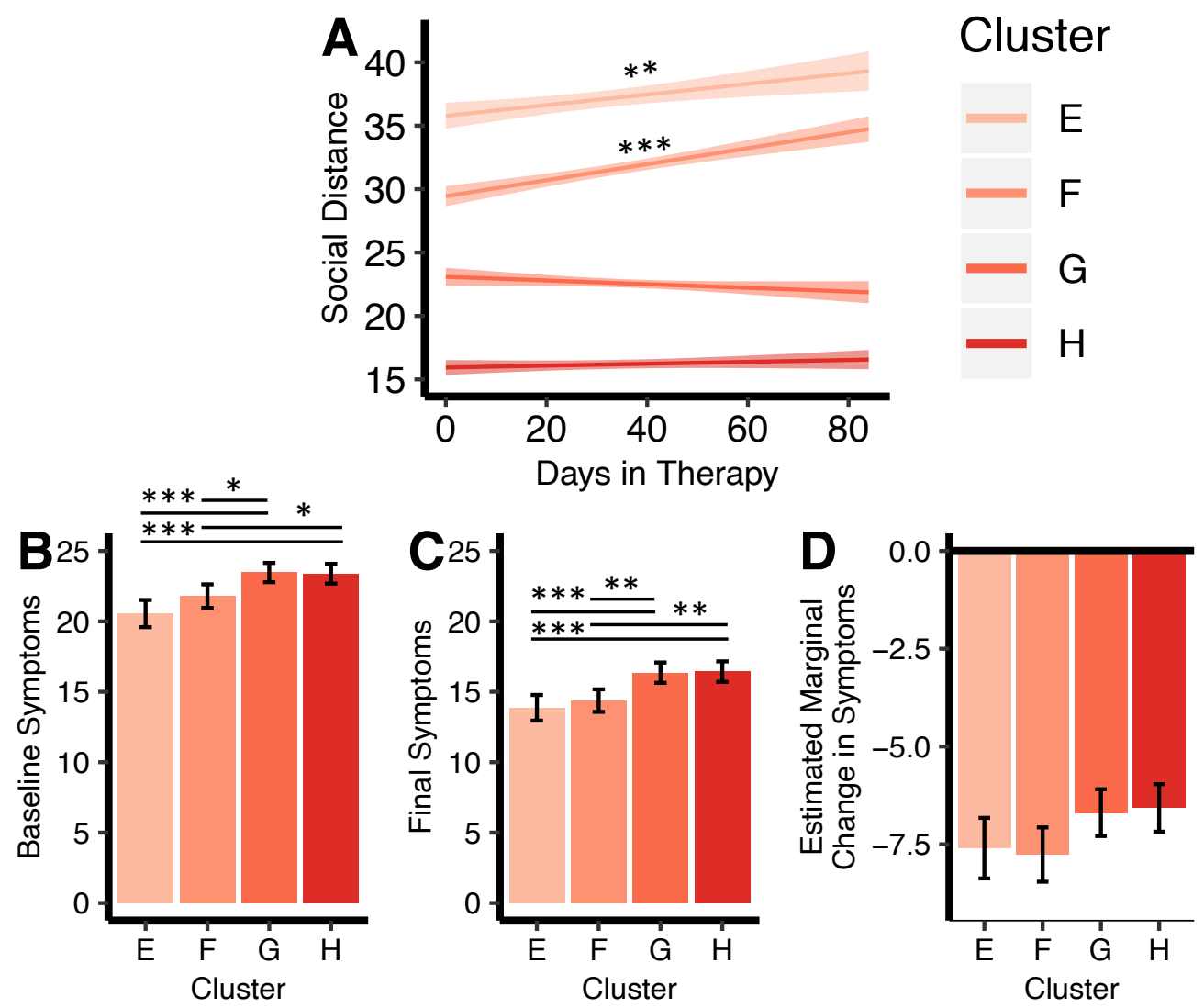

Figure S4. Results of the mixture regression clustering participants in the validation dataset based on social distance. (A) Four clusters were identified, with cluster $\mathrm{E}_{\mathrm{v}}$ starting high and increasing over time $\beta_{\mathrm{v}}=.09$, $p_{\mathrm{v}}=.002, R^{2} \beta_{\mathrm{v}}=.04, N_{\mathrm{v}}=453$, cluster $\mathrm{F}_{\mathrm{v}}$ starting slightly lower and increasing over time, $\beta_{\mathrm{v}}=.17, p_{\mathrm{v}}<.001$, $R^{2} \beta_{v}=.08, N_{v}=560$, cluster $G_{v}$ starting lower and not significantly changing over time, $\beta_{v}=-.04, p_{v}=.097$, $R^{2} \beta_{v}=.004, N_{v}=757$, and cluster $\mathrm{H}_{\mathrm{v}}$ starting the lowest and not significantly changing over time, $\beta_{\mathrm{v}}=.02$, $p_{\mathrm{v}}=.289, R^{2}{ }_{3 v}=.002, N_{\mathrm{v}}=730$. (B) Clusters differed significantly in baseline internalizing symptoms, with clusters $E$ and $F$ reporting significantly fewer symptoms than clusters $G$ and $H$. (C) Clusters also differed significantly in final internalizing symptoms, with clusters $E$ and $F$ reporting significantly fewer symptoms than clusters $G$ and $H$. (D) Clusters did not differ significantly in their average symptom change over the course of therapy. Results replicate the exploratory dataset. ${ }^{* *} p<.001,{ }^{* *} p<.01,{ }^{*} p<.05$. 


\section{Supplementary References}

1. Nook EC, Schleider JL, Somerville LH (2017) A linguistic signature of psychological distancing in emotion regulation. J Exp Psychol Gen 146(3):337-346.

2. Nook EC, Vidal Bustamante CM, Cho HY, Somerville LH (2020) Use of linguistic distancing and cognitive reappraisal strategies during emotion regulation in children, adolescents, and young adults. Emotion 20(4):525-540.

3. Shahane AD, Denny BT (2019) Predicting emotional health indicators from linguistic evidence of psychological distancing. Stress Heal 35(2):200-210.

4. Pennebaker JW, Booth RJ, Francis ME (2007) Linguistic Inquiry and Word Count: LIWC [Computer software].

5. American Psychiatric Association (2013) Diagnostic and Statistical Manual of Mental Disorders (Author, Washington, DC). 5th Ed.

6. Bernstein EE, et al. (2019) Unique and predictive relationships between components of cognitive vulnerability and symptoms of depression. Depress Anxiety 36(10):950-959.

7. Joormann J, Talbot L, Gotlib IH (2007) Biased processing of emotional information in girls at risk for depression. J Abnorm Psychol 116(1):135-43.

8. Prinstein MJ, Borelli JL, Cheah CSL, Simon V a, Aikins JW (2005) Adolescent girls' interpersonal vulnerability to depressive symptoms: a longitudinal examination of reassurance-seeking and peer relationships. J Abnorm Psychol 114(4):676-88.

9. Abramson LY, et al. (1999) Cognitive vulnerability to depression: Theory and evidence. J Cogn Psychother An Int Q 13(1):5-20.

10. Hankin BL, Abramson LY (2002) Measuring cognitive vulnerability to depression in adolescence: Reliability, validity, and gender differences. J Clin Child Adolesc Psychol 31(4):491-504.

11. Kircanski K, Thompson RJ, Sorenson JE, Sherdell L, Gotlib IH (2015) Rumination and worry in daily life: Examining the naturalistic validity of theoretical constructs. Clin Psychol Sci 3(6):926939.

12. Nolen-Hoeksema S, Wisco BE, Lyubomirsky S (2008) Rethinking rumination. Perspect Psychol Sci 3(5):400-424.

13. McEvoy PM, Watson H, Watkins ER, Nathan P (2013) The relationship between worry, rumination, and comorbidity: Evidence for repetitive negative thinking as a transdiagnostic construct. J Affect Disord 151(1):313-320.

14. Adler JM, Harmeling LH, Walder-Biesanz I (2013) Narrative meaning making is associated with sudden gains in psychotherapy clients' mental health under routine clinical conditions. $J$ Consult Clin Psychol 81(5):839-845. 